\title{
OPTIMAL DESIGN OF NONLINEAR ENERGY SINKS FOR SDOF STRUCTURES SUBJECTED TO WHITE NOISE BASE EXCITATIONS
}

\author{
Maria Oliva $^{1}$, Giorgio Barone ${ }^{2}$ and Giacomo Navarra ${ }^{1}$
}

${ }^{1}$ Faculty of Engineering and Architecture, University of Enna Kore, Cittadella Universitaria, Enna, Italy

${ }^{2}$ School of Civil and Building Engineering, Loughborough University, Loughborough, Leicestershire, UK

\begin{abstract}
Nonlinear Energy Sinks (NESs) have recently received increasing attention from researchers because of their capability to passively absorb a significant amount of energy over a wide range of frequencies. In most studies, the dynamic response of the main structure coupled with one or more NESs is analysed for impulsive loading. In this paper, the performance of the NES attached to a Single Degree of Freedom (SDOF) system, under random Gaussian white noise base excitations, is investigated through several numerical simulations. In order to determine the optimal configuration for the device, four different objective functions are considered. Sensitivity analyses with respect to the intensity of the random loads, the mass ratio and the main parameters of the primary structure are presented. The authors propose an approximate design approach based on the use of the Statistical Linearization Technique, and an accurate empirical formulation linking the NES optimal parameters to the characteristic of the main structure and the random excitation. Numerical results are validated by Monte Carlo simulations. Finally, a numerical application for a 2-DOFs system equipped with a NES has been presented in order to investigate the applicability of the proposed empirical approach for Multi Degrees of Freedom structures.
\end{abstract}

Author keywords: Nonlinear Energy Sink; Passive Control; Optimisation; Structural dynamics; White Noise; Statistical Linearization Technique. 


\section{INTRODUCTION}

The growing urbanization and the constant advances in building technologies have led to increasingly tall, slender and lightweight structures, often vulnerable to dynamic loads (e.g. wind and earthquakes). In the last decades several control strategies [1] have been developed to protect tall structures against environmental actions, thus reducing structural vibrations. Dynamic vibration absorbers belong to the class of passive control systems. These devices have been widely used for civil engineering structures because of their capability to operate without requiring an external power source. This implies simplicity in design, maintenance and operation. However, conventional linear dynamic vibration absorbers are generally tuned to one of the modal frequencies of the main structure in order to suppress the contributions from fundamental modes. Consequently, they are very sensitive to the detuning, caused for example by creep, temperature effects or significant variation of the structural parameters.

The Nonlinear Energy Sink (NES) is able to resonate with any mode of the primary structure because of its nonlinear nature. It is, therefore, insensitive to frequency changes and more robust than linear Tuned Mass Dampers (TMDs) [2]. The NES consists of a small mass coupled to the main structure with a nonlinear spring and a damping element. It is capable to passively and irreversibly absorb and dissipate a significant amount of energy from the primary structure over a wide range of frequencies. This one-way irreversible transfer, known as passive target energy transfer, occurs in systems of damped oscillators mainly via transient resonance capture and has been widely investigated both analytically [3-8] and experimentally [9-14]. NES configurations can be categorized depending on the operating mechanisms, as grounded $[3,4]$ or ungrounded $[5,6]$, and as smooth $[7,15,16]$ or nonsmooth $[9,10]$. Specifically, a grounded configuration consists of an essentially nonlinear grounded oscillator coupled to the primary structure through a weakly linear stiffness, whereas in an ungrounded one the NES is directly and strongly coupled to the primary structure through the essentially nonlinear stiffness; a smooth NES configuration involves a cubic nonlinear stiffness, whereas a non-smooth one involves nonlinearities in the form of clearances or vibro-impacts. The performance of these types of NES has been numerically studied and experimentally tested under different loading conditions, such as impulsive [6,14,16,17], periodic [18-21], seismic [10,13,14,22] and stationary random loadings [2].

Despite the high capacity of this nonlinear absorber to reduce the dynamic response of the structures, the presence of the nonlinear stiffness term makes the NESs especially sensitive to loading perturbations. Therefore, the optimal design of the device parameters is of the utmost importance. Several optimisation criteria and objective functions have been introduced in literature, depending on the characteristics of the main system and the external excitations. In [23,24], a procedure to obtain optimal design of the energetic sinks with amplitude-phase variables has been proposed by considering the analytical solution of energy pumping problem for strongly nonhomogeneous twoDegree of Freedom (DOF) systems. A closed-form solution for the nonlinear stiffness of the NES bounds of stability has been proposed in reference [25], whereas an analytical tuning for the nonlinear stiffness of the NES under transient and harmonic excitations has been proposed in [26]. In [27], optimal design criteria to determine the minimum nonlinear stiffness required to activate the target energy transfer have been proposed for transient regime. A reliability-based design optimisation of the NES has been presented in reference [28] for the cases of two- and three- DOFs systems, under impulsive loading. Genetic Algorithms have been used to find the optimal parameters of NESs in order to mitigate the vibrations of beams subjected to moving loads in [29]. 
The performance of a NES coupled to a linear damped Eulero-Bernoulli beam has been also considered in [30], where an optimisation procedure has been performed for various boundary conditions with two different values of forcing amplitude. A two-DOFs structure, subjected to a bandlimited white noise, has been investigated in [2]. In the latter study, the authors have shown that the optimal nonlinear stiffness decreases for increasing amplitude of the load. Moreover, the performance of the NES increases with the natural frequency of the main structure. However, these results have been obtained considering only one sample of white noise.

In this paper, the optimal parameters of a smooth ungrounded NES have been investigated for the case of a structure subjected to random base excitations, modelled as samples of a random Gaussian white noise process. The aim of this work is to analyse the dependence of the optimal NES parameters on the main structure characteristics and on the intensity of the random excitation in order to define a simplified optimisation procedure.

First, a simplified approach based on the Statistical Linearization Technique (SLT) has been used to obtain an approximate formula for the optimal nonlinear stiffness of the NES. The formula has been determined minimising the mean square error between the nonlinear system and the linearised one, subjected both to the same Gaussian excitation. The results have been compared with Monte Carlo simulations, in order to determine the error committed in evaluating the optimal parameters.

Then, a parametric analysis has been performed in order to investigate the dependence of the NES performance on the main characteristics of the system by varying the intensity of the random loading, the mass ratio between the NES and the primary structure, as well as the frequency and the damping ratio of the main structure. The numerical results are critically discussed, focusing on the sensitivity of the NES optimal nonlinear stiffness and linear damping. Empirical relationships among the optimal parameters, the amplitude of the load and the main system parameters are proposed for pre-design purposes and validated against the results of Monte Carlo simulations. Moreover, a simplified design of NESs for MDOF systems which involves the application of these empirical formulae is presented through a numerical application on a 2DOF model.

\section{STRUCTURAL MODEL}

A schematic diagram of the system considered herein is shown in Figure 1. Known in literature as "Configuration II" [8] or "Type I" [22,31] NES, it consists of a SDOF primary structure connected to an ungrounded and lightweight NES through a pure cubic stiffness and a linear viscous damper. The equations of motion of the combined system can be written as following:

$$
\left\{\begin{array}{l}
m_{1} \ddot{x}_{1}+c_{1} \dot{x}_{1}+c_{2}\left(\dot{x}_{1}-\dot{x}_{2}\right)+k_{1} x_{1}+k_{2}\left(x_{1}-x_{2}\right)^{3}=-m_{1} \ddot{x}_{g} \\
m_{2} \ddot{x}_{2}+c_{2}\left(\dot{x}_{2}-\dot{x}_{1}\right)+k_{2}\left(x_{2}-x_{1}\right)^{3}=-m_{2} \ddot{x}_{g}
\end{array}\right.
$$

in which $m_{1}, c_{1}, k_{1}$ and $x_{1}$ are mass, viscous damping coefficient, stiffness coefficient and displacement relative to the base of the main structure, respectively; $m_{2}, c_{2}, k_{2}$ and $x_{2}$ are mass, viscous damping coefficient, nonlinear stiffness coefficient and displacement relative to the base of the NES, respectively; dots mean derivative with respect to time, and $\ddot{x}_{g}$ is the base acceleration modelled as a zero-mean stationary Gaussian white noise, having Power Spectral Density (PSD) amplitude $S_{0}$.

Dividing Eq. (1) by $m_{1}$, the nonlinear system of equations becomes: 
in which:

$$
\lambda_{1}=\frac{c_{1}}{m_{1}}=2 \zeta_{1} \omega_{1} ; \quad \lambda_{2}=\frac{c_{2}}{m_{1}} ; \quad \omega_{1}^{2}=\frac{k_{1}}{m_{1}} ; \quad \kappa=\frac{k_{2}}{m_{1}} ; \quad \varepsilon=\frac{m_{2}}{m_{1}} .
$$

where $\zeta_{1}$ and $\omega_{1}$ are the damping ratio and the frequency of the primary structure and $\varepsilon$ is the mass ratio between the NES and the main structure. Several papers showed that even simple systems may exhibit complex dynamics involving fundamental and sub-harmonic resonances, nonlinear beating phenomena and multi-frequency responses $[5,6,8]$.

An interesting feature of the NES is its ability to passively and irreversibly absorb and locally dissipate a significant amount of vibrational energy from the primary structures. Moreover, the NES is capable to interact over broad frequency bands, thus its performance is not significantly affected by structural frequency changes and it is more robust than linear passive absorbers. However, the NES performance is critically dependent on the amplitude of the external excitation since it was demonstrated that it only works properly for specific ranges of forcing amplitudes. This is due to the fact that the response of nonlinear systems is not proportional to the amplitude excitation, as it occurs in linear ones. Therefore, the performance of a nonlinear system optimised for a specific load condition can vary significantly for a different load.

\section{OPTIMAL NES PARAMETERS}

Although there are several papers discussing the NES and the nonlinear energy pumping phenomenon, however only few of them aim to design optimal NES parameters for given primary system specifications. This is due to the complex dynamic behaviour of the nonlinear system and the NES sensitivity to loading perturbations that make particularly difficult designing the optimal parameters of this device. Closed-form solutions to determine the optimal nonlinear stiffness of the NES capable to trigger the targeted energy transfer have been proposed only for transient and harmonic excitations [25-27], whereas, to the authors' knowledge, there are no analytical or numerical formulae for white noise excitations.

Of course, the definition of the optimal NES parameters depends by the selected criteria used to estimate the effectiveness of the NES itself. Herein, these criteria have been chosen among those introduced in $[32,33]$ and widely used to optimise different NES configurations $[2,9,10,34]$. Since in this work the focus is on the protection of the main structure by reducing its response, the objective functions have been defined as the dimensionless ratio between a selected characteristic of the dynamic response of the primary structure in its controlled (i.e. with attached NES) and uncontrolled (i.e. removing the NES) configurations, respectively. Therefore, these objective functions represent different measures of the NES performance (the lower the value of each objective function, the higher the NES performance) and their minimisation leads to determining the optimal NES parameters.

The first two objective functions consider the ratios of the Root Mean Square (RMS) of the displacements and absolute accelerations of the controlled and uncontrolled systems, respectively, i.e. they are defined as: 


$$
\hat{J}_{1}=\frac{R M S\left(x_{1, C}(t)\right)}{R M S\left(x_{1, U}(t)\right)}
$$

$$
\hat{J}_{2}=\frac{R M S\left(\ddot{x}_{1 a, C}(t)\right)}{R M S\left(\ddot{x}_{1 a, U}(t)\right)}
$$

in which $x_{1, C}$ and $\ddot{x}_{1 a, C}$ are the displacements and the absolute accelerations of the main structure of the controlled system, while $x_{1, U}$ and $\ddot{x}_{1 a, U}$ are the displacements and the absolute accelerations of the uncontrolled system, respectively.

Two additional objective functions have been defined as the ratios of the maximum displacements and absolute accelerations between the controlled and the uncontrolled systems:

$$
\begin{aligned}
& \hat{J}_{3}=\frac{\max \left(x_{1, C}(t)\right)}{\max \left(x_{1, U}(t)\right)} \\
& \hat{J}_{4}=\frac{\max \left(\ddot{x}_{1 a, C}(t)\right)}{\max \left(\ddot{x}_{1 a, U}(t)\right)}
\end{aligned}
$$

The choice to minimise the dynamic response in terms of both displacements and accelerations was made in order to take into account the structural integrity and safety of the structure, on one hand, and the functionality of non-structural elements and comfort of occupants, on the other.

\subsection{Case Study}

In order to compare the four proposed objective functions, a case study has been selected as reference structure based on a real small-scale experimental test [35]. The main structure is realised connecting two steel columns through two nylon rigid plates, one of which is directly attached to a shaking table. All the details of the primary structure are reported in Table 1. The cubic nonlinear stiffness is realised by adopting the configuration proposed in reference [11]. The NES, attached to the top storey of the system, is constituted by a small mass able to slide on a guide by means of a low friction car and connected to the structure through a steel wire, with no pretension, located perpendicularly to the mass motion direction.

In this first analysis, a mass ratio $\varepsilon=0.05$ between the NES and the primary structure has been considered. Gaussian random white noise samples, 20 seconds long, have been used as accelerations to the base of the structure. It has been observed that increasing the duration of the base motion time histories does not significantly affect the results.

A numerical searching technique has been used to identify the optimal values of the nonlinear stiffness $\kappa$ and the damping $\lambda_{2}$ of the NES. Monte Carlo Simulations (MCS) have been performed using, for each analysis, 10000 base acceleration time histories with temporal sampling equal to $0.01 \mathrm{~s}$ and modelled as samples of a Gaussian random white noises with a constant PSD function with amplitude $S_{0}=10^{-3}\left(\mathrm{~m} / \mathrm{s}^{2}\right)^{2} /(\mathrm{rad} / \mathrm{s})$, and directly integrating the nonlinear equations of motion by the 4th-order Runge-Kutta method. Each objective function, named $J_{i}(i=1, \ldots, 4)$, has been obtained as the average of the functions $\hat{J}_{i}(i=1, \ldots, 4)$, defined in eqs. (3)-(6), over the 10000 samples. A preliminary analysis has been conducted to assess the accuracy of the MCS. 10000 samples have been 
selected providing an estimated variation of the average results smaller than $0.1 \%$. Figures $2 \mathrm{a}-\mathrm{b}$ report a sample of the white noise process and the one-side PSD of the white noise process $\left(G_{0}=2 \cdot S_{0}\right)$ reproduced using 10000 samples, respectively.

The variations of the four proposed objective functions with respect to the parameters $\kappa$ and $\lambda_{2}$ are shown in Figure 3. A well-defined region of high effectiveness of the NES can be easily identified for all cases. In fact, despite the values of the objective functions are different, the positions of their minima in the search space are approximately the same in all four cases. Table 2 summarises the optimal NES parameters for each objective function. Among them, the second one results the most sensitive to variations of the parameters $\kappa$ and $\lambda_{2}$. However, since the position of the minima is about the same, in all the following analyses only $J_{1}$ will be considered in order to guarantee mainly the structural integrity.

Because of the nonlinear behaviour of the coupled system, the numerical results of the optimisation are strongly dependent on the selected structure and intensity of the excitation. In the following, two methodologies are proposed to directly determine the optimal NES configuration. The first approach is based on the Statistical Linearization Technique (SLT) allowing to derive two analytic, although approximate formulae for the optimal NES parameters. The second one, instead, proposes high accuracy empirical formulae based on the numerical results of a campaign of MCSs on several different structures and white noise intensities.

\section{OPTIMISATION OF THE NES PARAMETERS BY SLT}

In this section, an approximate expression for the optimal NES parameters is obtained by linearizing the system through the SLT and optimising it based on the linear TMD theory. Proposed firstly by Caughey in reference [36], the SLT is an effective method to apply random vibration analysis on systems with hysteretic nonlinear restoring forces [37]. In the last decades, approaches based on the SLT have been proposed to study the performance of different passive control systems under random loads [38-47].

For the nonlinear system described in section 2, the equations of motion (2) can be rewritten in compact form as:

$$
\mathbf{M} \ddot{\mathbf{X}}(t)+\mathbf{C} \dot{\mathbf{X}}(t)+\mathbf{K X}(t)+\mathbf{g}(\mathbf{X}(t))=-\mathbf{M} \boldsymbol{\tau} \ddot{X}_{g}(t)
$$

where $\mathbf{X}(t)=\left[\begin{array}{ll}X_{1}(t) & X_{2}(t)\end{array}\right]^{\mathrm{T}}$ and the capital letters indicate stochastic processes; $\mathbf{M}, \mathbf{C}$ and $\mathbf{K}$ are the mass, damping and linear stiffness matrices of the combined system, respectively:

$$
\mathbf{M}=\left[\begin{array}{ll}
1 & 0 \\
0 & \varepsilon
\end{array}\right] ; \quad \mathbf{C}=\left[\begin{array}{cc}
\lambda_{1}+\lambda_{2} & -\lambda_{2} \\
-\lambda_{2} & \lambda_{2}
\end{array}\right] ; \quad \mathbf{K}=\left[\begin{array}{cc}
\omega_{1}^{2} & 0 \\
0 & 0
\end{array}\right]
$$

$\boldsymbol{\tau}=\left[\begin{array}{ll}1 & 1\end{array}\right]^{\mathrm{T}}$ is the location vector; and $\mathbf{g}(\mathbf{X}(t))$ is the following nonlinear vector:

$$
\mathbf{g}(\mathbf{X}(t))=\kappa\left[\begin{array}{l}
\left(X_{1}(t)-X_{2}(t)\right)^{3} \\
\left(X_{2}(t)-X_{1}(t)\right)^{3}
\end{array}\right]
$$


As shown in the previous section, the optimal parameters of the NES have been determined minimising also the objective function $J_{1}$, defined in terms of RMS of the displacements of the nonlinear system. This can be estimated, according to the SLT [48], by replacing the initial nonlinear system with the following equivalent linear one:

$$
\mathbf{M} \ddot{\mathbf{X}}(t)+\mathbf{C} \dot{\mathbf{X}}(t)+\left(\mathbf{K}+\mathbf{K}_{e}\right) \mathbf{X}(t)=-\mathbf{M} \boldsymbol{\tau} \ddot{X}_{g}(t)
$$

in which $\mathbf{K}_{e}$ is the equivalent linear stiffness matrix, defined as:

$$
\mathbf{K}_{e}=\varepsilon \omega_{2, e}^{2}\left[\begin{array}{rr}
1 & -1 \\
-1 & 1
\end{array}\right]
$$

The normalised equivalent linear stiffness $\varepsilon \omega_{2, e}^{2}$ can be determined minimising the mean square error committed replacing eq. (7) with eq. (10), i.e.

$$
E\left[\left(\mathbf{g}(\mathbf{x}(t))-\mathbf{K}_{e} \mathbf{x}(t)\right)^{T}\left(\mathbf{g}(\mathbf{x}(t))-\mathbf{K}_{e} \mathbf{x}(t)\right)\right] \rightarrow \min _{\varepsilon \omega_{2, e}^{2}}
$$

where $E[\cdot]$ indicates the ensemble average. By minimising this error, the normalised equivalent stiffness is obtained as:

$$
\varepsilon \omega_{2, e}^{2}=3 \kappa E\left[\left(X_{2}(t)-X_{1}(t)\right)^{2}\right]
$$

Since the response of the equivalent linear system excited by a zero-mean Gaussian process is a zero-mean Gaussian process itself, eq. (13) can be rewritten as:

$$
\omega_{2, e}^{2}=3 \frac{\kappa}{\varepsilon} \sigma_{X_{2}-X_{1}}^{2}=3 \frac{\kappa}{\varepsilon} \sigma_{Y}^{2}
$$

where $\sigma_{Y}^{2}$ is the variance of the relative displacements $Y=X_{2}-X_{1}$ between the control device and the main structure in the linearised system, and it can be computed as:

$$
\sigma_{Y}^{2}=\sigma_{X_{1}}^{2}+\sigma_{X_{2}}^{2}-2 \sigma_{X_{1} X_{2}}
$$

Since the base excitation is a white noise process, the steady-state variances can be determined in closed-form by equating to zero the Lyapunov equation of the evolution of the covariance matrix [43]:

$$
\mathbf{D}_{s} \boldsymbol{\Sigma}_{\mathbf{Z}}(t)+\boldsymbol{\Sigma}_{\mathbf{Z}}(t) \mathbf{D}_{s}^{\mathrm{T}}+\mathbf{G}_{s} \mathbf{G}_{s}^{T} \pi S_{0}=\dot{\boldsymbol{\Sigma}}_{\mathbf{Z}}(t)=\mathbf{0}
$$

where $\boldsymbol{\Sigma}_{\mathbf{Z}}(t)$ is the covariance matrix in terms of the state-space vector $\mathbf{Z}=\left[\begin{array}{ll}\mathbf{X} & \dot{\mathbf{X}}\end{array}\right]^{\mathrm{T}}, \mathbf{D}_{s}$ and $\mathbf{G}_{s}$ are defined as:

$$
\mathbf{D}_{s}=\left[\begin{array}{cc}
\mathbf{0} & \mathbf{I}_{2} \\
-\mathbf{M}^{-1}\left(\mathbf{K}+\mathbf{K}_{e}\right) & -\mathbf{M}^{-1} \mathbf{C}
\end{array}\right] ; \quad \mathbf{G}_{s}=\left[\begin{array}{c}
\mathbf{0} \\
\mathbf{M}^{-1} \boldsymbol{\tau}
\end{array}\right]
$$

and $\mathbf{I}_{2}$ is the 2 by 2 identity matrix. Eq. (16) leads to the following expression: 


$$
\sigma_{Y}^{2}=\frac{\pi S_{0} \omega_{1}}{2 \omega_{2, e}^{3} z}
$$

in which the factor $z$ is defined as:

$$
z=\frac{\zeta_{1} \zeta_{2} \omega_{1}^{4}+\left(\varepsilon+4 \zeta_{1}^{2}\right) \zeta_{2}^{2} \omega_{1}^{3} \omega_{2, e}+2 \zeta_{1} \zeta_{2}\left(-1+2 \zeta_{1}^{2}+2(1+\varepsilon) \zeta_{2}^{2}\right) \omega_{1}^{2} \omega_{2, e}^{2}+\zeta_{1}^{2}\left(\varepsilon+4(1+\varepsilon) \zeta_{2}^{2}\right) \omega_{1} \omega_{2, e}^{3}+(1+\varepsilon)^{2} \zeta_{1} \zeta_{2} \omega_{2, e}^{4}}{\zeta_{1} \omega_{1}^{3}+\left(\varepsilon+4 \zeta_{1}^{2}\right) \zeta_{2} \omega_{1}^{2} \omega_{2, e}+\zeta_{1}\left(\varepsilon+4 \zeta_{1}^{2}+4(1+\varepsilon) \zeta_{2}^{2}\right) \omega_{1} \omega_{2, e}^{2}+(1+\varepsilon)\left(1+\varepsilon+4 \zeta_{1}^{2}\right) \zeta_{2} \omega_{2, e}^{3}}
$$

By substituting eq. (18) into eq. (14), the normalised NES nonlinear stiffness $\kappa$ can be written as a function of the linearised one as:

$$
\kappa=\frac{2 \varepsilon \omega_{2, e^{5}}^{5}}{3 \pi S_{0} \omega_{1}}
$$

The linear damping coefficient of the NES is instead equal to the damping of the linearised system:

$$
\lambda_{2}=2 \zeta_{2} \varepsilon \omega_{2, e}
$$

Eqs. (20) and (21) can be exploited to determine the optimal NES design by using the linear TMD theory, for which closed-form optimal solutions have been proposed in literature [49-51], depending on the selected optimisation criteria, on the characteristics of the main structure and on the loading conditions. The analytic solution proposed by Asami et al. [50] has been selected to optimise the equivalent linear system for the case study introduced in section 3.1. This solution has been obtained for random excitations, minimising the area under the frequency response function, i.e. the variance of the displacements of the main structure. Hence, if the variances of the linear and nonlinear systems are equal, Asami's optimisation criterion coincides with minimising the squared value of $J_{1}$. The solutions selected for the determination of the optimum damping ratio $\zeta_{2, \text { opt }}$ and the optimum frequency ratio $v_{\text {opt }}$ are:

$$
\zeta_{2, o p t}=-\frac{\zeta_{1}}{v_{\text {opt }}} \frac{c_{0}+c_{1} \zeta_{1}^{2}+c_{2} \zeta_{1}^{4}+c_{3} \zeta_{1}^{6}+c_{4} \zeta_{1}^{8}+c_{5} \zeta_{1}^{10}+c_{6} \zeta_{1}^{12}}{d_{0}+d_{2} \zeta_{1}^{4}+d_{3} \zeta_{1}^{6}+d_{4} \zeta_{1}^{8}+d_{5} \zeta_{1}^{10}+c_{6} \zeta_{1}^{12}}
$$

$$
v_{\text {opt }}=\frac{\omega_{2, o p t}}{\omega_{1}}=\sqrt{-\frac{p_{2}}{2}-\sqrt{\frac{p_{2}^{2}}{4}-q_{2}}}
$$

in which the first parameter is function of the structural damping, mass ratio and optimum frequency ratio, while the second one only depends on structural damping and mass ratio. For the determination of the coefficient $c_{i}, d_{i}, p_{2}$ and $q_{2}$, readers should refer to [50]. Finally, substituting eqs. (22) and (23) into (19)-(21), the optimal NES parameters are easily determined.

The accuracy of the results obtained using the proposed procedure has been assessed by comparison with MCSs. In Table 3, the optimal NES stiffness and damping, normalised with respect to the structural mass, are listed for assigned structural damping ratio $\left(\zeta_{1}=\zeta_{1, R}\right)$ and excitation amplitude $\left(S_{0}=10^{-3}\left(\mathrm{~m} / \mathrm{s}^{2}\right)^{2} /(\mathrm{rad} / \mathrm{s})\right)$ and for three different structural frequencies. The error 
committed in estimating the NES optimal parameters has been computed using the following expressions:

$$
\begin{aligned}
& e_{\log _{10} \kappa}=\frac{\left|\log _{10}\left[\kappa_{o p t, M C S}\right]-\log _{10}\left[\kappa_{o p t, S L T}\right]\right|}{\left|\log _{10}\left[\kappa_{o p t, M C S}\right]\right|} \\
& e_{\lambda_{2}}=\frac{\left|\lambda_{2, \text { opt }, M C S}-\lambda_{2, o p t, S L T}\right|}{\left|\lambda_{2, o p t, M C S}\right|}
\end{aligned}
$$

where $\kappa_{o p t, M C S}$ and $\kappa_{o p t, S L T}$ are the normalised nonlinear stiffness obtained through Monte Carlo Simulations and through the formula (20), respectively; $\lambda_{2, o p t, M C S}$ and $\lambda_{2, o p t, S L T}$ are the normalised NES damping coefficients obtained through Monte Carlo Simulations and through the formula (21), respectively. As shown in Table 3, the error committed in the evaluation of the nonlinear stiffness (in logarithmic scale) is below $5 \%$, whereas for the NES damping is about $20 \%$.

Figure 4 shows the $J_{1}$ values for the nonlinear system having parameters determined by eqs. (22) and (23) versus the actual minimum of $J_{1}$, for three different values of the natural frequency of the main structure. The following error function has been considered:

$$
e_{J_{1}}=\frac{\left|J_{1, o p t, M C S}-J_{1, o p t, S L T}\right|}{\left|J_{1, \text { opt }, M C S}\right|}
$$

where $J_{1, \text { opt }, M C S}$ and $J_{1, \text { opt SLT }}$ are values of $J_{1}$ determined using the optimal NES parameters obtained through the MCS and SLT, respectively. It can be noted that this error is less than $2 \%$, as reported in Table 3, and this confirms the robustness of the NES.

Although the NES is characterised by a strongly nonlinear stiffness term, this section showed how the SLT approach still manages to return a quasi-optimal NES configuration in a stochastic sense. Moreover, the proposed approach leads to analytic (although approximate) formulae for both NES parameters, avoiding the computational effort required to perform the optimisation and Monte Carlo simulation described into the previous section.

\section{EMPIRICAL FORMULAE FOR OPTIMAL NES PARAMETERS}

Since the SLT-based approach does not exactly predict the optimal NES parameters, a parametric study has been conducted in order to obtain a more accurate design tool. In particular, the sensitivity of the NES performance with respect to the intensity of the random excitation, the structural frequency, the structural damping ratio and the mass ratio, has been analysed. The results have been assessed against the results of Monte Carlo simulations and compared with those obtained by the first proposed approach.

\subsection{Parametric Analysis}

Unlike linear vibration absorbers, the effectiveness of the NES is dependent on the intensity and type of load on the main system. Hence, the optimal nonlinear stiffness, linking the device to the main structure, has to be re-determined for any change on the white noise PSD amplitude. 
Figure 5 shows how the objective function $J_{1}$ changes when the reference primary structure is excited by white noise excitations whose PSD amplitude ranges between $10^{-4}$ and $10^{-2}$ $\left(\mathrm{m} / \mathrm{s}^{2}\right)^{2} /(\mathrm{rad} / \mathrm{s})$. These values have been selected in order to generate base acceleration peaks ranging between $0.05 \mathrm{~g}$ and $1 \mathrm{~g}$, corresponding to typical ground accelerations for civil engineering applications. The general dynamic behaviour of the system does not change and a well-defined region of effectiveness for each case can be identified in all considered cases. Although the objective function maintains the same shape and value, it shifts in a direction parallel to the $\kappa$ axis. Increasing values of the amplitude of the PSD function of the white noise lead to decreasing optimal nonlinear stiffness, as also proposed in [2]. Conversely, the normalised optimal damping $\lambda_{2}$, equal to $0.276 \mathrm{~s}^{-1}$, is not affected by the change in PSD amplitude $S_{0}$.

The optimal nonlinear stiffness coefficients $\kappa$, marked with red dots in Figure 6a, have been numerically fitted using the following linear relationship (solid line) between the logarithms of the optimal value of $\kappa$ and $S_{0}$ :

$$
\log _{10} \kappa_{\text {opt }}=A \log _{10} S_{0}+B
$$

where $\kappa_{\text {opt }}$ is the normalised optimal nonlinear stiffness expressed in $(\mathrm{m} \mathrm{s})^{-2}$ and the coefficients $A$ and $B$, determined considering $S_{0}$ in $\left(\mathrm{m} / \mathrm{s}^{2}\right)^{2} /(\mathrm{rad} / \mathrm{s})$, depend on the main structure parameters. For the selected case study, $A=-0.99\left(\mathrm{~s} / \mathrm{m}^{2}\right)^{2}(\mathrm{rad} / \mathrm{s})$ and $B=2.52(\mathrm{~m} \mathrm{~s})^{-2}$ have been identified. Since the analytic formula for the nonlinear stiffness based on the SLT, eq. (20), shows an inverse proportionality between the nonlinear stiffness and the PSD amplitude, the value of $A$ has been rounded up to -1 . For the considered case, the maximum difference between the values obtained through numerical simulations and using eq. (27) is $0.04 \%$. Analogous results have been obtained for all the four proposed objective functions. Moreover, the authors verified that eq. (27) holds also for PSD amplitudes larger than those reported in this paper.

A sensitivity analysis has been carried out in order to quantify the effects of the variation of the primary structure parameters on the NES optimal design. The minimum $J_{1}$ has been determined for several configurations of the primary structure, varying its frequency $\omega_{1}$, damping ratio $\zeta_{1}$ and the mass ratio $\varepsilon$. The amplitude of the PSD function of the excitation process, $S_{0}$, has been varied as described previously.

Figures 6a-b report the optimal $\kappa_{\text {opt }}$ and $\lambda_{2, o p t}$ against $S_{0}$ for five different values of $\omega_{1}$ (selected with respect to the frequency of the reference structure, $\omega_{1, R}$ ) and $\varepsilon=0.05$. All the numerical results, marked with different symbols, follow eq. (27). The optimal nonlinear stiffness, in fact, decreases increasing $S_{0}$ while the optimal damping coefficient remains constant. Since fitting lines obtained by eq. (27) are parallel, then the coefficient $A$ is the same for any value of the main frequency $\omega_{1}$. Conversely, the coefficient $B$, increases for increasing $\omega_{1}$.

Analogously, the dependence of the optimal NES parameters on the structural damping ratio $\zeta_{1}$ (selected with respect to the damping ratio of the reference structure, $\zeta_{1, R}$ ) has been investigated, as shown in Figures 7a-b. Numerical results confirm again the validity of eq. (27). The coefficient $A$ is independent on the damping ratio $\zeta_{1}$ and the coefficient $B$ increases for increasing damping ratio of the primary structure.

Finally, Figures $8 \mathrm{a}-\mathrm{b}$ show the effects of the variation of the amplitude of the white noise excitation on the optimal parameters $\kappa_{\text {opt }}$ and $\lambda_{2, o p t}$ for different values of the mass ratio $\varepsilon$. The assumption of lightweight NES has been introduced not only to trigger the passive target energy transfer, that occurs 
for small values of the mass of the NES, but also for practical issues related to the costs and realisation of the system. For this reason, the values of the mass ratio $\varepsilon$ considered in the analysis have been set at $0.025,0.05$ and 0.1 . The optimal nonlinear stiffness, inversely proportional to the amplitude of the PSD function, increases also increasing the mass ratio.

With regards to the normalised optimal NES damping $\lambda_{2, \text { opt }}$, the latter increases increasing the main frequency of the primary structure as well as the mass ratio and decreasing the structural damping ratio, as shown in Figures $6 \mathrm{~b}, 7 \mathrm{~b}$ and $8 \mathrm{~b}$. The optimal NES parameters and the corresponding values of the $J_{1}$ are listed in Table 4 for the case of $\varepsilon=0.05$ and $S_{0}=10^{-3}\left(\mathrm{~m} / \mathrm{s}^{2}\right)^{2} /(\mathrm{rad} / \mathrm{s})$.

\subsection{Optimal nonlinear stiffness and damping of the NES}

Extending the parametric analysis to several combinations of $\omega_{1}, \zeta_{1}, S_{0}$ and $\varepsilon$, and fitting all the numerical results, the following empirical formulae for the nonlinear stiffness $\left[(\mathrm{m} \mathrm{s})^{-2}\right]$ and damping $\left[\mathrm{s}^{-1}\right]$ of the NES have been obtained:

$$
\log _{10} \kappa_{\text {opt }}=-\log _{10} S_{0}+4.98 \log _{10} \omega_{1}+0.21 \log _{10} \zeta_{1}+1.33 \log _{10} \varepsilon-1.908
$$

$$
\lambda_{2, \text { opt }}=(0.204 \varepsilon-0.001) \zeta_{1}^{-0.1} \omega_{1}
$$

where the coefficients have been determined considering $S_{0}$ expressed in $\left(\mathrm{m} / \mathrm{s}^{2}\right)^{2} /(\mathrm{rad} / \mathrm{s})$ and $\omega$ in $\mathrm{rad} / \mathrm{s}$.

Eqs. (28) and (29) are graphically represented in Figures 9a-c as function of frequency and damping ratio of the main structure. In particular, Figure 9a shows the dependence of the optimal nonlinear stiffness on the PSD function amplitude for $\varepsilon=0.05$, whereas Figure $9 \mathrm{~b}$ shows its dependence on the mass ratio for $S_{0}=10^{-3}\left(\mathrm{~m} / \mathrm{s}^{2}\right)^{2} /(\mathrm{rad} / \mathrm{s}) . \kappa_{\text {opt }}$ increases with the frequency and damping of the primary structure, yet it is more sensitive to the former than the latter.

The optimal damping ratio $\lambda_{2, \text { opt }}$ does not depend on the intensity of the excitation. Conversely, its dependence on the mass ratio is reported in Figure 9c. The NES damping is directly proportional to the structural frequency and mass ratio and decreases for increasing structural damping ratio.

Taking advantage of eqs. (28) and (29), optimal design charts can be created for the evaluation of the NES parameters. Examples are shown in Figures 10-12, for three values of the mass ratio. These diagrams allow to quickly determine the optimal NES parameters for assigned main structure (frequency and damping) and selected mass ratio and intensity of the random excitation.

The error (with respect to MCSs), committed estimating the optimal parameters and the function $J_{1}$ using the SLT-based approach and the proposed empirical formulae, has been determined varying the involved parameters $\left(\omega_{1}, \zeta_{1}, S_{0}\right.$ and $\varepsilon$ ). These errors are shown in Figures 13-15 for the case of mass ratio $\varepsilon=0.05$ and for three values of the PSD amplitude.

For the optimal nonlinear stiffness, the error increases with the PSD amplitude, however the absolute error does not change. The maximum error is obtained for $S_{0}=10^{-3}\left(\mathrm{~m} / \mathrm{s}^{2}\right)^{2} /(\mathrm{rad} / \mathrm{s})$ and it is equal to $16.84 \%$ using the SLT-based approach, while only $3.53 \%$ using the empirical one.

Otherwise, the errors in terms of $\lambda_{2, \text { opt }}$ and $J_{1}$ determined using both the proposed approaches are approximately the same for each values of $S_{0}$, as shown in Figures 14-15. In fact, the NES damping and the minimum value of $J_{1}$ do not depend on the amplitude of the excitation, as previously shown in Figure 5. For the optimal NES damping, the SLT-based approach can produce significant errors, with 
a maximum error (for the studied case) of $32.5 \%$. Conversely, by using the empirical formula the error is below $5.5 \%$ for all the values of structural frequency and damping ratio except for the case of $\omega_{1}=4 \omega_{1, R}$ and $\zeta_{1}=2 \zeta_{1, R}$ in which the error is $13.9 \%$. Finally, the maximum error in terms of $J_{1}$ is $4 \%$ for the SLT-based approach, versus only $0.86 \%$ for the empirical approach.

Although it is not statistically significant, a sample of the response process of the reference structure (Table 1) is presented in Figures 16a-b in order to show the effectiveness of the optimised NES with the proposed empirical formulae (28) and (29). In particular, a comparison between the uncontrolled (i.e. removing the NES) and controlled (i.e. with attached NES) configurations, in terms of relative displacements to the base (Figure 16a) and of absolute accelerations (Figure 16b) of the primary structure is showed. Initially, the NES does not work properly because it undergoes small oscillations and most of the input energy is localised to the primary structure. The reduction of the response occurs when the level of the motion increases, i.e. when the motion is strongly localised in the NES thus activating the energy pumping from the primary system, as shown in Figures 16c-d. Furthermore, Figures 16e-f report the comparison between the two configurations in the frequencydomain in terms of the PSD of the response process. These show that the NES is an effective passive control device and it is capable to guarantee the requirements of structural integrity and comfort.

\section{SIMPLIFIED DESIGN OF NESs FOR MULTI-DOF STRUCTURES: A NUMERICAL APPLICATION}

Although the numerical formulae presented in this work have been obtained only on the base of SDOF models, they can be adopted for MDOF structures for an approximate simplified design of NESs. In this section, the dynamic response of a 2-DOF linear system coupled with a NES, optimised by means of eqs. (28) and (29), has been investigated. A schematic diagram of this system is reported in Figure 17. A case study has been selected based on a 2-DOF physical experimental model utilised in the Smart Structure Technology Laboratory at the University of Illinois at Urbana-Champaign [2,14,52]. The lumped masses are 24.3 and $24.2 \mathrm{~kg}$ for the first and second storeys, respectively; the stiffnesses are 6820 and $8220 \mathrm{~N} / \mathrm{m}$ for the first and second storeys, respectively, and the damping of the model is set at $2 \%$ at each mode. The resulting natural frequencies of this structure are 10.62 and $29.09 \mathrm{rad} / \mathrm{s}$ for the first and second modes, respectively. The equations of motion of the 2-DOF structure in its uncontrolled configuration are:

$$
\left\{\begin{array}{l}
m_{1} \ddot{x}_{1}+c_{1} \dot{x}_{1}+k_{1} x_{1}+c_{2}\left(\dot{x}_{1}-\dot{x}_{2}\right)+k_{2}\left(x_{1}-x_{2}\right)=-m_{1} \ddot{x}_{g} \\
m_{2} \ddot{x}_{2}+c_{2}\left(\dot{x}_{2}-\dot{x}_{1}\right)+k_{2}\left(x_{2}-x_{1}\right)=-m_{2} \ddot{x}_{g}
\end{array}\right.
$$

in which $m_{i}, c_{i}, k_{i}$ and $x_{i}(i=1,2)$ are respectively mass, viscous damping coefficient, stiffness coefficient and displacement relative to the base of the $i$-th storey, and $\ddot{x}_{g}$ is the base acceleration modelled as a zero-mean stationary Gaussian white noise, with PSD amplitude $S_{0}$. The equations of motion of the 2-DOF system combined with an ungrounded NES (i.e. controlled configuration) can be written as:

$$
\left\{\begin{array}{l}
m_{1} \ddot{x}_{1}+c_{1} \dot{x}_{1}+k_{1} x_{1}+c_{2}\left(\dot{x}_{1}-\dot{x}_{2}\right)+k_{2}\left(x_{1}-x_{2}\right)=-m_{1} \ddot{x}_{g} \\
m_{2} \ddot{x}_{2}+c_{2}\left(\dot{x}_{2}-\dot{x}_{1}\right)+k_{2}\left(x_{2}-x_{1}\right)+c_{N}\left(\dot{x}_{2}-\dot{x}_{N}\right)+k_{N}\left(x_{2}-x_{N}\right)^{3}=-m_{2} \ddot{x}_{g} \\
m_{N} \ddot{x}_{N}+c_{N}\left(\dot{x}_{N}-\dot{x}_{2}\right)+k_{N}\left(x_{N}-x_{2}\right)^{3}=-m_{N} \ddot{x}_{g}
\end{array}\right.
$$


where $m_{N}, c_{N}, k_{N}$ and $x_{N}$ are mass, viscous damping coefficient, stiffness coefficient and displacement relative to the base of the NES.

The design of the NES has been carried out by considering a SDOF structure with mass equivalent to the effective modal mass at the first mode of the original structure $\left(m_{1, e}=46.65 \mathrm{~kg}\right)$, frequency $\omega_{1}=10.62 \mathrm{rad} / \mathrm{s}$ (the first modal frequency) and damping $2 \%$. Therefore, applying eqs. (28) and (29) for $S_{0}=10^{-3}\left(\mathrm{~m} / \mathrm{s}^{2}\right)^{2} /(\mathrm{rad} / \mathrm{s}), \omega_{1}=10.62 \mathrm{rad} / \mathrm{s}, \zeta_{1}=0.02$ and $\varepsilon=0.05$, the NES optimal parameters has been directly determined, i.e.:

$$
\begin{aligned}
& m_{N}=0.05 \cdot m_{1, e}=2.33 \mathrm{~kg} \\
& k_{N}=\kappa_{\text {opt }} \cdot m_{1, e}=10^{5.78} \mathrm{~N} / \mathrm{m}^{3} \\
& c_{N}=\lambda_{2, o p t} \cdot m_{1, e}=6.74 \mathrm{Ns} / \mathrm{m}
\end{aligned}
$$

The accuracy of the results has been assessed by comparison with MCSs. The latter have been performed using 10000 base acceleration time histories, modelled as samples of a Gaussian random white noise and directly integrating the nonlinear equations of motion by the 4th-order Runge-Kutta method. Two objective functions $J_{1}$ and $J_{2}$, based on the ratio of the RMS of the displacements of the two storeys in their controlled and uncontrolled configurations, have been selected for the optimisation problem. These have been obtained by averaging the following functions over the generated 10000 samples:

$$
\begin{aligned}
& \hat{J}_{1}=\frac{R M S\left(x_{1, C}(t)\right)}{R M S\left(x_{1, U}(t)\right)} \\
& \hat{J}_{2}=\frac{R M S\left(x_{2, C}(t)\right)}{R M S\left(x_{2, U}(t)\right)}
\end{aligned}
$$

in which $\hat{J}_{1}$ and $\hat{J}_{2}$ are defined for a single sample of base acceleration time-history; $x_{1, C}$ and $x_{2, C}$ are the displacements of the first and second floors of the controlled system, while $x_{1, U}$ and $x_{2, U}$ are the displacements of the first and second floors of the uncontrolled system, respectively.

Figures $18 \mathrm{a}-\mathrm{b}$ show $J_{1}$ and $J_{2}$ values for the nonlinear system having parameters determined by eqs. (28) and (29) (marked with red dots) versus the actual minima of $J_{1}$ and $J_{2}$ obtained by MCSs (marked with black dots). It can be noted that the error committed on the reduction of the RMS of the displacements of the primary structure is less than $1 \%$.

The dampening effects of the NES (optimised by using the proposed empirical formulation) are shown, for a single sample of the response of the system in its controlled and uncontrolled configurations, in terms of displacements of the two storeys (Figures 19a-b). Since the optimisation approach is defined in a stochastic framework, results in the frequency-domain are shown in terms of the PSD of the 10000 samples of response determined by the MCS (Figure 19c-d), instead of just the Fourier spectrum of the response of a single sample. The comparison in the frequency-domain in terms of PSD of response process shows a considerable reduction of the response at the first mode.

The proposed simplified optimisation procedure for the NES works well for MDOF systems dominated by one mode, as, for example, in the case of high-rise buildings. Hence, the higher the effective modal mass at that mode, the better the performance of the NES is. Further investigation on 
the interaction between modes and on the most appropriate objective functions to minimise is required to overcome this limitation.

\section{CONCLUSIONS}

439

In this paper, the optimal design of an ungrounded NES attached to a SDOF structure excited by a stationary Gaussian white noise process has been investigated. Two design approaches have been presented in order to reduce the computational burden required to perform a numerical optimisation.

In the first case, an analytic procedure based on the Statistical Linearization Technique and the TMD theory has been proposed. The optimal nonlinear stiffness has been obtained by minimising the mean square error between the nonlinear system and the linearised one with respect to its structural parameters. Accordingly, a relationship between the parameters of the NES and its linear equivalent system has been determined. The latter acts as a TMD, whose optimal parameters (i.e. those minimising the RMS of the response of the main structure) are known in closed-form in literature. Hence, by inverse process, the NES optimal parameters are determined by first opportunely tuning its equivalent system, and then exploiting the relationships between the structural parameters of both linear and nonlinear systems. Although this method does not predict the exact optimal NES parameters, it returns a quasi-optimal design of the NES. For the analysed case study, the maximum error estimated on the reduction of the RMS of the displacements of the primary structure (used as a measure of the NES effectiveness) does not exceed 3.6\%.

The second approach is fully numerical and provides empirical formulae that can be straightforwardly used to determine the optimal NES parameters. For this purpose, a parametric study with respect to the intensity of the random excitation, the structural frequency, the structural damping ratio and the mass ratio, has been performed using Monte Carlo Simulations. By a curve fitting procedure of the optimal NES parameters obtained for several configurations of the main system, empirical formulae for the optimal NES stiffness and damping have been derived for any primary structure and any random load intensity. These formulae allow for a significant reduction of the error committed in the evaluation of the optimal parameters of the NES with respect to the first approach. The easy implementation of the formulae entails also a reduced computational effort, making the method suitable for practical engineering purposes. With this aim, it has been shown how the proposed empirical formulae can be easily translated in optimal NES design charts.

The sensitivity of the optimal NES parameters with respect to the above mentioned parameters has been widely investigated. In particular, it has been demonstrated that the optimal nonlinear stiffness is inversely proportional to the amplitude of the PSD function of the white noise, yet it increases with the parameters of the main structure (frequency and damping ratio) as well as the mass ratio between the NES and the primary structure. The optimal NES damping, instead, is independent from the amplitude of the white noise, increases for increasing structural frequency and mass ratio and for decreasing damping ratio of the primary structure.

Finally, the applicability of the proposed approach to MDOF structures has been shown through a numerical application on a 2DOF model. The optimal NES parameters in this case are obtained by considering an equivalent SDOF structure, whose structural parameters are based on the effective modal mass at the first mode of vibrations. The proposed design methodology works well for MDOF 
systems dominated by one mode (e.g. high-rise buildings) and further studies are required to overcome this limitation.

\section{REFERENCES}

480

[1] Housner GW, Bergman LA, Caughey TK, Chassiakos AG, Claus RO, Masri SF, et al. Structural Control : Past, Present and Future. J Eng Mech 1997;123:897-971.

[2] Wierschem NE, Spencer Jr BF, Bergman LA, Vakakis AF. Numerical study of nonlinear energy sinks for seismic response reduction. Proc. 6th Int. Work. Adv. Smart Mater. Smart Struct. Technol., 2011.

[3] Gendelman O, Manevitch LI, Vakakis AF, M'Closkey R. Energy pumping in nonlinear mechanical oscillators: Part I-Dynamics of the underlying hamiltonian systems. J Appl Mech 2001;68:34.

[4] Vakakis AF, Gendelman OV. Energy pumping in nonlinear mechanical oscillators: Part II-Resonance Capture. J Appl Mech 2001;68:42.

[5] Kerschen G, Lee YS, Vakakis A., McFarland DM, Bergman LA. Irreversible passive energy transfer in coupled oscillators with essential nonlinearity. SIAM J Appl Math 2005;66:648-79.

[6] Lee YS, Kerschen G, Vakakis AF, Panagopoulos P, Bergman L, McFarland DM. Complicated dynamics of a linear oscillator with a light, essentially nonlinear attachment. Phys D Nonlinear Phenom 2005;204:41-69.

[7] Gendelman OV. Transition of energy to a nonlinear localized mode in a highly asymmetric system of two oscillators. Nonlinear Dyn 2001;25:237-53.

[8] Vakakis AF, Gendelman OV, Bergman LA, McFarland DM, Kerschen G, Lee YS. Nonlinear targeted energy transfer in mechanical and structural systems. vol. 156. Dordrecht: Springer Netherlands; 2009.

[9] Nucera F, Vakakis AF, McFarland DM, Bergman LA, Kerschen G. Targeted energy transfers in vibroimpact oscillators for seismic mitigation. Nonlinear Dyn 2007;50:651-77.

[10] Nucera F, Lo Iacono F, McFarland DM, Bergman LA, Vakakis AF. Application of broadband nonlinear targeted energy transfers for seismic mitigation of a shear frame: Experimental results. J Sound Vib 2008;313:57-76.

[11] McFarland DM, Bergman LA, Vakakis AF. Experimental study of non-linear energy pumping occurring at a single fast frequency. Int J Non Linear Mech 2005;40:891-9.

[12] Kerschen G, McFarland DM, Kowtko JJ, Lee YS, Bergman LA, Vakakis AF. Experimental demonstration of transient resonance capture in a system of two coupled oscillators with essential stiffness nonlinearity. J Sound Vib 2007;299:822-38.

[13] Gourdon E, Alexander NA, Taylor CA, Lamarque CH, Pernot S. Nonlinear energy pumping under transient forcing with strongly nonlinear coupling: Theoretical and experimental results. J Sound Vib 2007;300:522-51.

[14] Wang J, Wierschem NE, Spencer BF, Lu X. Track Nonlinear energy sink for rapid response reduction in building structures. J Eng Mech 2015;141:4014104.

[15] Lee YS, Vakakis AF, Bergman LA, McFarland DM, Kerschen G, Nucera F, et al. Passive non-linear targeted energy transfer and its applications to vibration absorption: a review. Proc. Inst. Mech. Eng. Part K J. Multi-body Dyn., vol. 222, 2008, p. 77-134.

[16] Vakakis AF. Inducing passive nonlinear energy sinks in vibrating systems. J Vib Acoust 2001;123:324..

[17] Wang J, Wierschem N, Spencer BF, Lu X. Experimental study of track nonlinear energy sinks for dynamic response reduction. Eng Struct 2015;94:9-15.

[18] Gourc E, Michon G, Seguy S, Berlioz A. Experimental investigation and design optimization of targeted energy transfer under periodic forcing. J Vib Acoust 2014;136:21021.

[19] Jiang X, McFarland DM, Bergman LA, Vakakis AF. Steady state passive nonlinear energy pumping in coupled oscillators: theoretical and experimental results. Nonlinear Dyn 2003;33:87-102.

[20] Gendelman OV, Gourdon E, Lamarque CH. Quasiperiodic energy pumping in coupled oscillators under periodic forcing. J Sound Vib 2006;294:651-62.

[21] Starosvetsky Y, Gendelman OV. Response regimes of linear oscillator coupled to nonlinear energy sink with harmonic forcing and frequency detuning. J Sound Vib 2008;315:746-65.

[22] Luo J, Wierschem NE, Hubbard SA, Fahnestock LA, Quinn DD, McFarland DM, et al. Large-scale experimental evaluation and numerical simulation of a system of nonlinear energy sinks for seismic mitigation. Eng Struct 2014;77:34-48.

[23] Manevitch L., Gourdon E, Lamarque CH. Towards the design of an optimal energetic sink in a strongly inhomogeneous two-degree-of-freedom system. J Appl Mech 2007;74:1078. 
[24] Manevitch LI, Gendelman O, Musienko AI, Vakakis AF, Bergman L. Dynamic interaction of a semiinfinite linear chain of coupled oscillators with a strongly nonlinear end attachment. Phys D Nonlinear Phenom 2003;178:1-18.

[25] Starosvetsky Y, Gendelman O V. Attractors of harmonically forced linear oscillator with attached nonlinear energy sink. II: Optimization of a nonlinear vibration absorber. Nonlinear Dyn 2008;51:47-57.

[26] Vaurigaud B, Ture Savadkoohi A, Lamarque CH. Targeted energy transfer with parallel nonlinear energy sinks. Part I: Design theory and numerical results. Nonlinear Dyn 2011;66:763-80.

[27] Nguyen TA, Pernot S. Design criteria for optimally tuned nonlinear energy sinks — part 1: transient regime. Nonlinear Dyn 2012;69:1-19.

[28] Boroson E, Missoum S. Reliability-based design optimization of nonlinear energy sinks. 11th World Congr. Struct. Multidiscip. Optim., 2015, p. 750-7.

[29] Younesian D, Nankali A, Motieyan E. Optimal nonlinear energy sinks in vibration mitigation of the beams traversed by successive moving loads. J Solid Mech 2011;3:323-31.

[30] Parseh M, Dardel M, Ghasemi MH. Investigating the robustness of nonlinear energy sink in steady state dynamics of linear beams with different boundary conditions. Commun Nonlinear Sci Numer Simul 2015;29:50-71.

[31] Wierschem NE, Luo J, Hubbard S, Fahnestock LA, Spencer, Jr. BF, Vakakis AF, et al. Experimental Testing of a Large 9-Story Structure Equipped with Multiple Nonlinear Energy Sinks Subjected to an Impulsive Loading. Struct. Congr. 2013, 2013, p. 2241-52

[32] Spencer, Jr. BF, Christenson RE, Dyke SJ. Next generation benchmark control problem for seismically excited buildings. Proc. Second World Conf. Struct. Control, vol. 2, New York: Wiley; 1999, p. 11351360.

[33] Ohtori Y, Christenson RE, Spencer BF, Dyke SJ. Benchmark control problems for seismically excited nonlinear buildings. J Eng Mech 2004:366-85.

[34] Nucera F, McFarland DM, Bergman LA, Vakakis AF. Application of broadband nonlinear targeted energy transfers for seismic mitigation of a shear frame: Computational results. J Sound Vib 2010;329:2973-94.

[35] Lo Iacono F. Passive control strategies for seismically excited structures. Theoretical and experimental analisys. PhD Thesis, Mediterranean University of Reggio Calabria, Reggio Calabria, Italy, 2008.

[36] Caughey TK. Equivalent linearization techniques. J Acoust Soc Am 1963;35:1706-11.

[37] Spanos PD. Stochastic linearization in structural dynamics. Appl Mech Rev 1981;34:1-8.

[38] Pandey MD. Stochastic analysis of structures with passive seismic control. Can J Civ Eng 1995;22:97080.

[39] Won AYJ, Pires JA, Haroun MA. Performance assessment of tuned liquid column dampers under random seismic loading. Int J Non Linear Mech 1997;32:745-58.

[40] Yalla SK, Kareem A. Optimum absorber parameters for tuned liquid column dampers. J Struct Eng 2000;126:906-15.

[41] Socha L. Linearization in analysis of nonlinear stochastic systems: recent results—Part I: Theory. Appl Mech Rev 2005;58:178.

[42] Socha L. Linearization in analysis of nonlinear stochastic systems, recent results-Part II: applications. Appl Mech Rev 2005;58:303.

[43] Di Matteo A, Lo Iacono F, Navarra G, Pirrotta A. Direct evaluation of the equivalent linear damping for TLCD systems in random vibration for pre-design purposes. Int J Non Linear Mech 2014;63:19-30.

[44] Di Matteo A, Lo Iacono F, Navarra G, Pirrotta A. Optimal tuning of tuned liquid column damper systems in random vibration by means of an approximate formulation. Meccanica 2015;50:795-808.

[45] Guo AX, Xu XL, Wu B. Seismic reliability analysis of hysteretic structure with viscoelastic dampers. Eng Struct 2002;24:373-83.

[46] Di Paola M, La Mendola L, Navarra G. Stochastic seismic analysis of structures with nonlinear viscous dampers. J Struct Eng 2007;133:1475-8.

[47] Di Paola M, Navarra G. Stochastic seismic analysis of MDOF structures with nonlinear viscous dampers. Struct Control Heal Monit 2009;16:303-18.

[48] Atalik TS, Utku S. Stochastic linearization of multi-degree-of-freedom non-linear systems. Earthq Eng Struct Dyn 1976;4:411-20.

[49] Warburton GB. Optimum absorber parameters for minimizing vibration response. Earthq Eng Struct Dyn 1981;9:251-62.

[50] Asami T, Nishihara O, Baz AM. Analytical Solutions to $\mathrm{H}_{\infty}$ and $\mathrm{H}_{2}$ Optimization of Dynamic Vibration Absorbers Attached to Damped Linear Systems. J Vib Acoust 2002;124:284.

[51] Ghosh A, Basu B. A closed-form optimal tuning criterion for TMD in damped structures. Struct Control 
Heal Monit 2007;14:681-92.

591

[52] Wierschem NE, Quinn DD, Hubbard SA, Al-Shudeifat MA, McFarland DM, Luo J, et al. Passive damping enhancement of a two-degree-of-freedom system through a strongly nonlinear two-degree-offreedom attachment. J Sound Vib 2012;331:5393-407. 
Table 1: Characteristics of the reference main structure.

\begin{tabular}{cccccc}
\hline $\boldsymbol{m}_{1, \boldsymbol{R}}$ & $\boldsymbol{c}_{\mathbf{1 , \boldsymbol { R }}}$ & $\boldsymbol{k}_{\mathbf{1 , \boldsymbol { R }}}$ & $\boldsymbol{f}_{\mathbf{1 , \boldsymbol { R }}}$ & $\boldsymbol{\omega}_{\mathbf{1 , \boldsymbol { R }}}$ & $\boldsymbol{\zeta}_{\mathbf{1 , \boldsymbol { R }}}$ \\
\hline$[\mathrm{Ns} / \mathrm{m}]$ & {$[\mathrm{Ns} / \mathrm{m}]$} & {$[\mathrm{N} / \mathrm{m}]$} & {$[\mathrm{Hz}]$} & {$[\mathrm{rad} / \mathrm{s}]$} & {$[\%]$} \\
\hline 2.135 & 1.57 & 890 & 3.25 & 20.42 & 1.8 \\
\hline
\end{tabular}


Table 2: Optimal parameters of the NES coupled to the reference main structure for $S_{0}=10^{-3}\left(\mathrm{~m} / \mathrm{s}^{2}\right)^{2} /(\mathrm{rad} / \mathrm{s})$.

\begin{tabular}{ccc}
\cline { 2 - 3 } & $\lambda_{2, o p t}$ & $\boldsymbol{\kappa}_{\text {opt }}$ \\
\cline { 2 - 3 } & {$\left[\mathrm{s}^{-1}\right]$} & {$\left[(\mathrm{s} \mathrm{m})^{-2}\right]$} \\
\hline $\boldsymbol{J}_{\mathbf{1}}$ & 0.276 & $10^{5.52}$ \\
\hline $\boldsymbol{J}_{2}$ & 0.278 & $10^{5.60}$ \\
\hline $\boldsymbol{J}_{\mathbf{3}}$ & 0.29 & $10^{5.52}$ \\
\hline $\boldsymbol{J}_{\mathbf{4}}$ & 0.29 & $10^{5.60}$ \\
\hline
\end{tabular}


Table 3: Comparison between the optimal parameters obtained through the MCS and through the SLT for $S_{0}=10^{-3}$ $\left(\mathrm{m} / \mathrm{s}^{2}\right)^{2} /(\mathrm{rad} / \mathrm{s})$.

\begin{tabular}{|c|c|c|c|c|c|c|c|c|c|c|}
\hline & \multirow{2}{*}{\multicolumn{4}{|c|}{$M C S$}} & \multirow{2}{*}{\multicolumn{3}{|c|}{ SLT }} & \multirow{2}{*}{\multicolumn{3}{|c|}{ Errors }} \\
\hline & & & & & & & & & & \\
\hline$\omega_{1}$ & $\zeta_{1}$ & $\lambda_{2, o p t}$ & $\log _{10}\left[\kappa_{\text {opt }}\right]$ & $J_{1}$ & $\lambda_{2, o p t}$ & $\log _{10}\left[\kappa_{\text {opt }}\right]$ & $J_{1}$ & $e_{\lambda_{2}}$ & $e_{\log _{10} \kappa}$ & $e_{J_{1}}$ \\
\hline$[\mathrm{rad} / \mathrm{s}]$ & [\%] & {$\left[\mathrm{s}^{-1}\right]$} & {$\left[(\mathrm{s} \mathrm{m})^{-2}\right]$} & {$[-]$} & {$\left[\mathrm{s}^{-1}\right]$} & {$\left[(\mathrm{s} \mathrm{m})^{-2}\right]$} & {$[-]$} & {$[\%]$} & {$[\%]$} & [\%] \\
\hline $2 \omega_{1, R}$ & $\zeta_{1, R}$ & 0.55 & 7.02 & 0.649 & 0.43 & 6.83 & 0.659 & 21.82 & 2.71 & 1.54 \\
\hline$\omega_{1, R}$ & $\zeta_{1, R}$ & 0.276 & 5.52 & 0.664 & 0.22 & 5.32 & 0.675 & 20.29 & 3.62 & 1.66 \\
\hline $0.5 \omega_{1, R}$ & $\zeta_{1, R}$ & 0.14 & 4.02 & 0.693 & 0.11 & 3.82 & 0.706 & 21.43 & 4.98 & 1.88 \\
\hline
\end{tabular}


Table 4: Optimal NES parameters for $S_{0}=10^{-3}\left(\mathrm{~m} / \mathrm{s}^{2}\right)^{2} /(\mathrm{rad} / \mathrm{s})$ and $\varepsilon=0.05$.

\begin{tabular}{|c|c|c|c|c|c|c|c|}
\hline & & & $0.25 \zeta_{1, R}$ & $0.5 \zeta_{1, R}$ & $\zeta_{1, R}$ & $2 \zeta_{1, R}$ & $4 \zeta_{1, R}$ \\
\hline \multirow{3}{*}{$0.25 \omega_{1, R}$} & $\lambda_{2, o p t}$ & {$\left[\mathrm{~s}^{-1}\right]$} & 0.08 & 0.075 & 0.069 & 0.064 & 0.060 \\
\hline & $\log _{10}\left[\kappa_{\text {opt }}\right]$ & {$\left[(\mathrm{s} \mathrm{m})^{-2}\right]$} & 2.40 & 2.50 & 2.53 & 2.62 & 2.69 \\
\hline & $J_{1}$ & {$[-]$} & 0.65 & 0.69 & 0.74 & 0.81 & 0.89 \\
\hline \multirow{3}{*}{$0.5 \omega_{1, R}$} & $\lambda_{2, o p t}$ & {$\left[\mathrm{~s}^{-1}\right]$} & 0.16 & 0.15 & 0.14 & 0.13 & 0.12 \\
\hline & $\log _{10}\left[\kappa_{o p t}\right]$ & {$\left[(\mathrm{s} \mathrm{m})^{-2}\right]$} & 3.90 & 3.96 & 4.02 & 4.09 & 4.13 \\
\hline & $J_{1}$ & {$[-]$} & 0.55 & 0.61 & 0.69 & 0.79 & 0.88 \\
\hline \multirow{3}{*}{$\omega_{1, R}$} & $\lambda_{2, o p t}$ & {$\left[\mathrm{~s}^{-1}\right]$} & 0.305 & 0.293 & 0.275 & 0.262 & 0.243 \\
\hline & $\log _{10}\left[\kappa_{o p t}\right]$ & {$\left[(\mathrm{s} \mathrm{m})^{-2}\right]$} & 5.40 & 5.46 & 5.52 & 5.58 & 5.65 \\
\hline & $J_{1}$ & {$[-]$} & 0.48 & 0.56 & 0.66 & 0.78 & 0.88 \\
\hline \multirow{3}{*}{$2 \omega_{1, R}$} & $\lambda_{2, o p t}$ & {$\left[\mathrm{~s}^{-1}\right]$} & 0.61 & 0.58 & 0.55 & 0.37 & 0.35 \\
\hline & $\log _{10}\left[\kappa_{o p t}\right]$ & {$\left[(\mathrm{s} \mathrm{m})^{-2}\right]$} & 6.93 & 6.96 & 7.02 & 7.08 & 7.16 \\
\hline & $J_{1}$ & {$[-]$} & 0.43 & 0.53 & 0.65 & 0.77 & 0.87 \\
\hline \multirow{3}{*}{$4 \omega_{1, R}$} & $\lambda_{2, o p t}$ & {$\left[\mathrm{~s}^{-1}\right]$} & 1.25 & 1.20 & 1.105 & 0.95 & 0.94 \\
\hline & $\log _{10}\left[\kappa_{\text {opt }}\right]$ & {$\left[(\mathrm{s} \mathrm{m})^{-2}\right]$} & 8.43 & 8.46 & 8.52 & 8.59 & 8.65 \\
\hline & $J_{1}$ & {$[-]$} & 0.47 & 0.56 & 0.66 & 0.78 & 0.87 \\
\hline
\end{tabular}




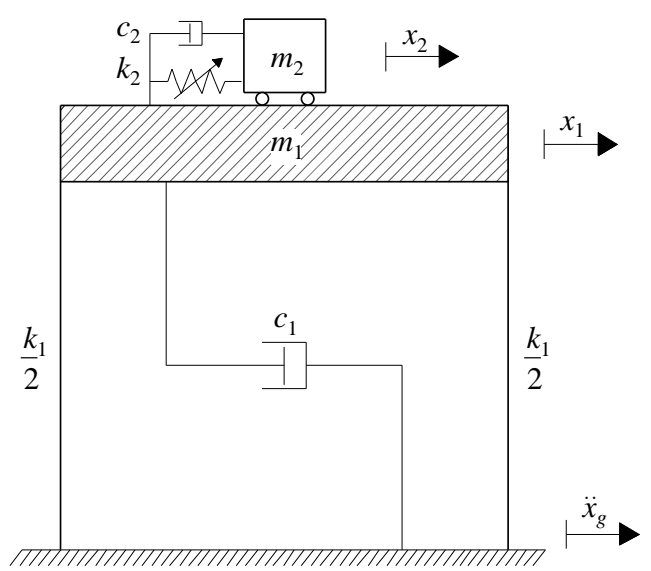

Figure 1: SDOF structure coupled with a NES. 
(a)

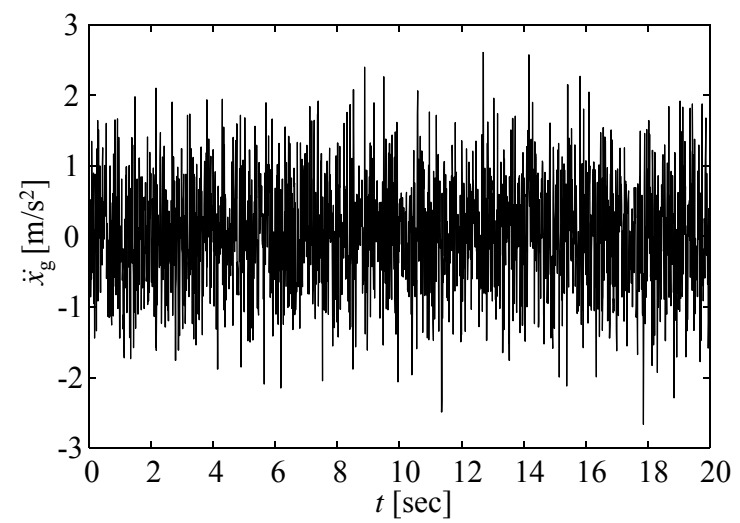

(b)

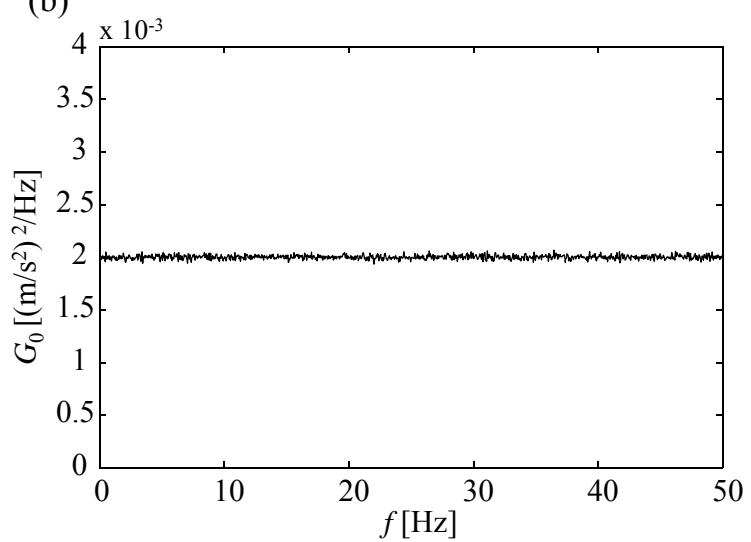

Figure 2: a) a sample of white noise process; b) one-side PSD of the white noise process. 
(a)

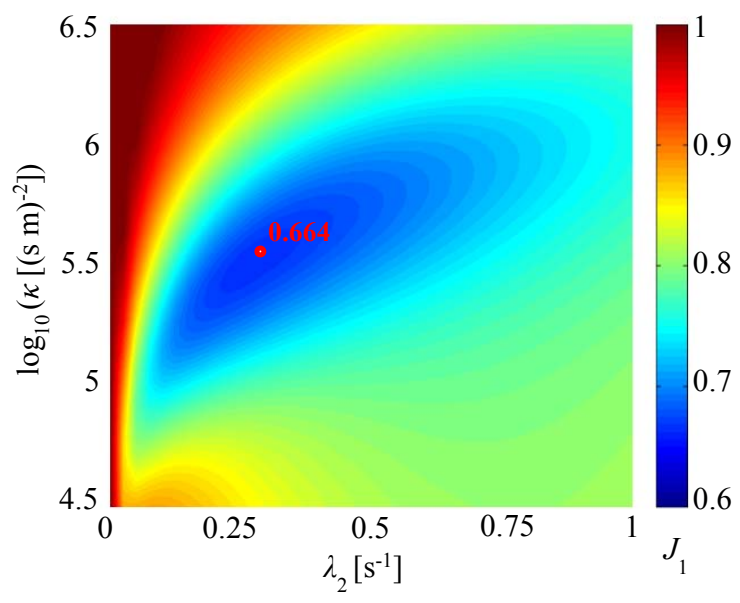

(c)

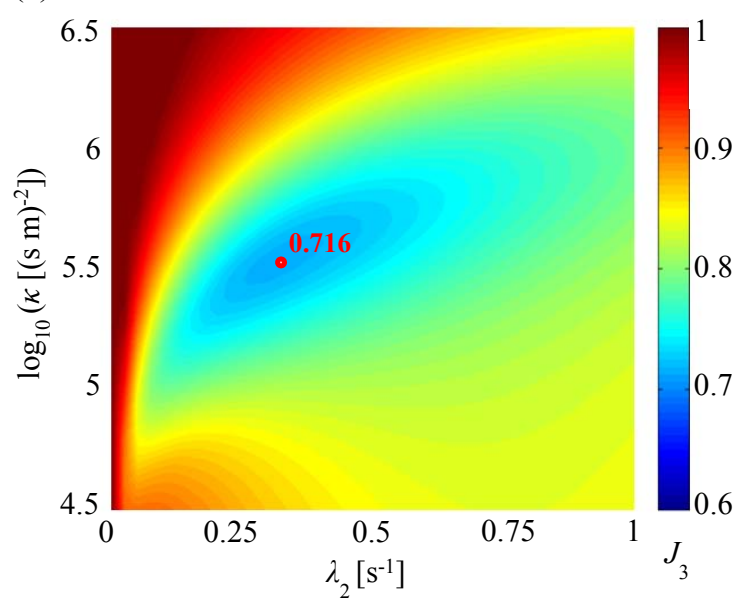

(b)

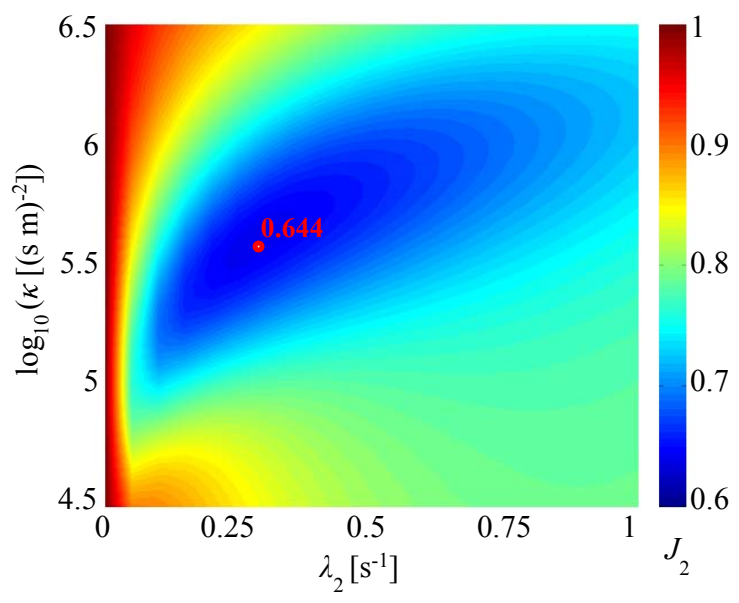

(d)

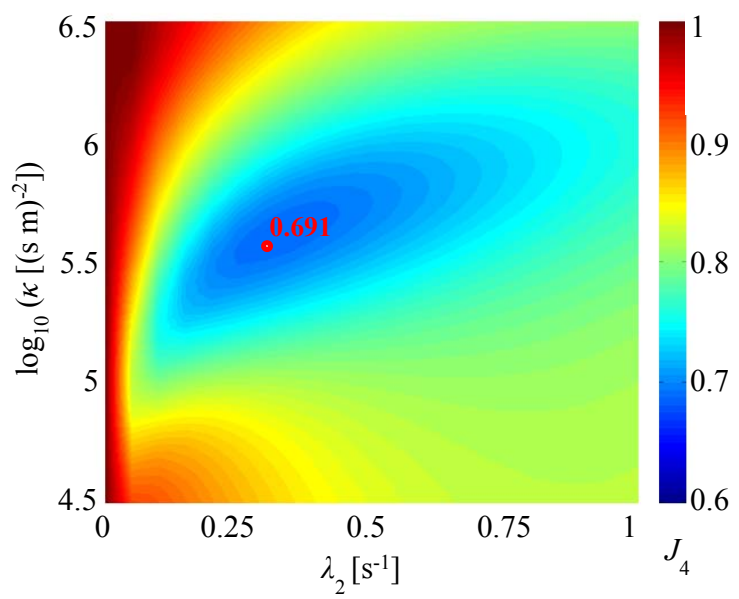

Figure 3: Objective functions with respect to the normalised NES parameters $\kappa$ and $\lambda_{2}$ : a) $J_{1}$; b) $J_{2}$; c) $J_{3}$; d) $J_{4}$. 
(a)

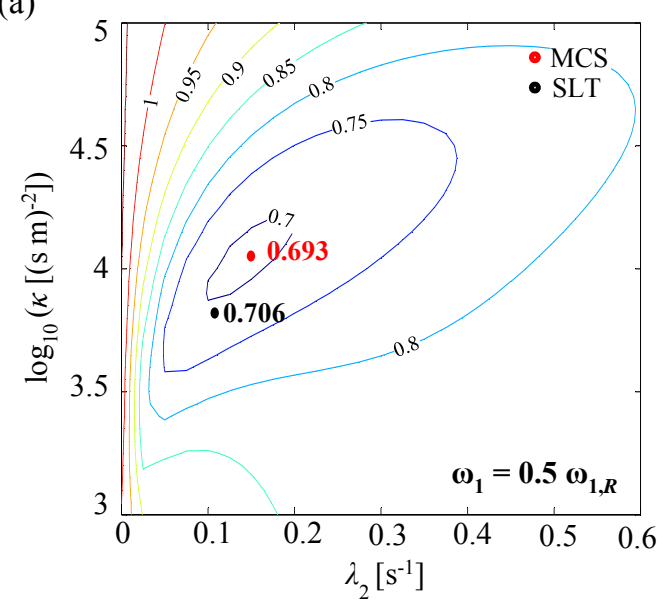

(b)

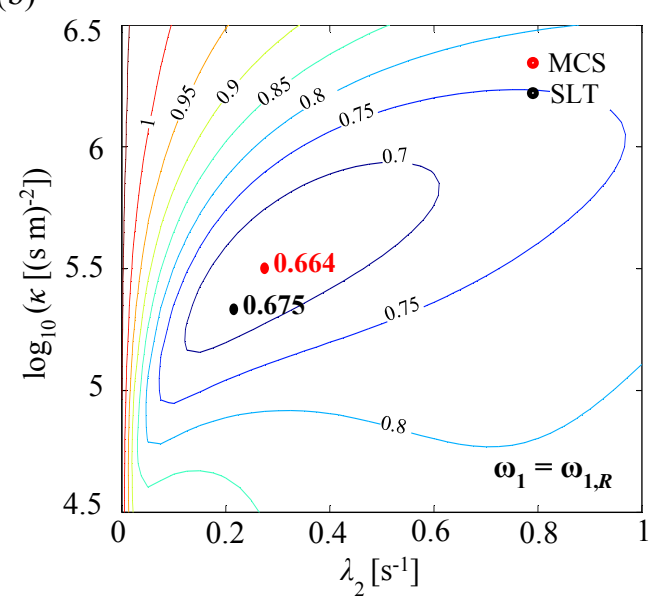

(c)

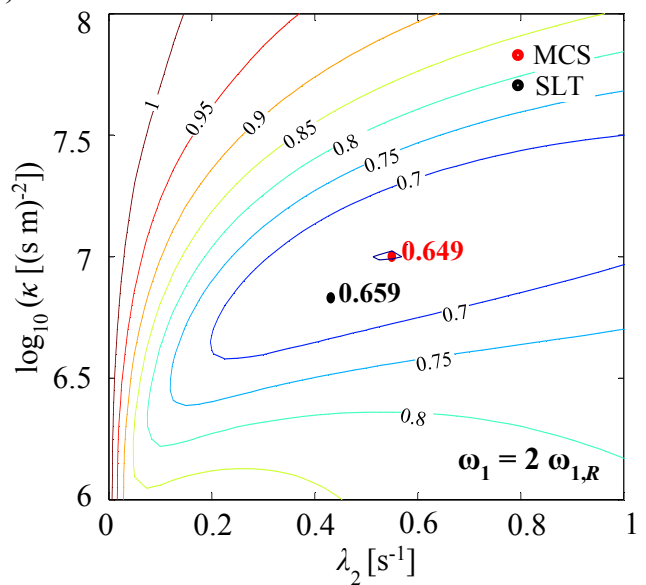

Figure 4: $J_{1}$ function and optimal NES parameters obtained by MCS (red dot) and SLT (black dot) for $S_{0}=10^{-3}\left(\mathrm{~m} / \mathrm{s}^{2}\right)^{2} /(\mathrm{rad} / \mathrm{s})$. 
(a)

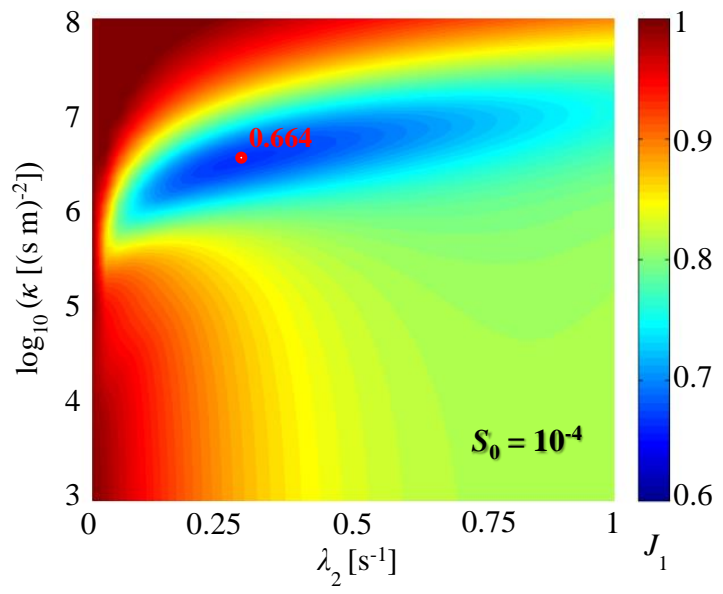

(c)

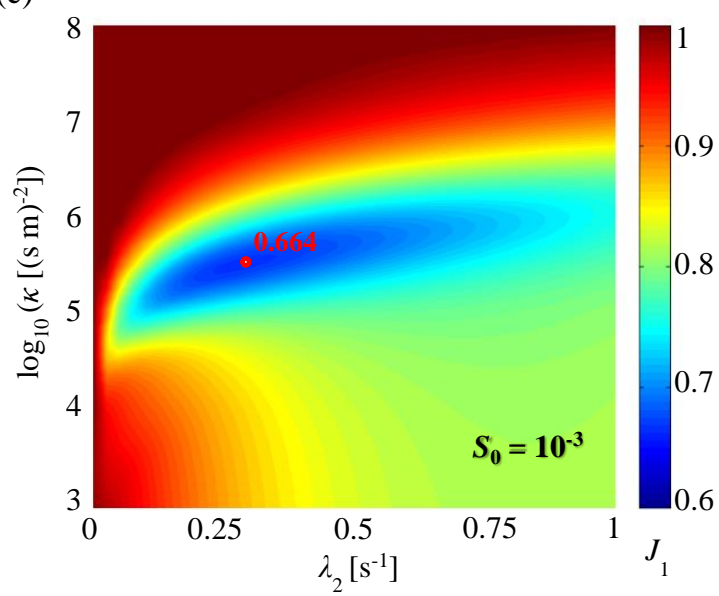

(b)

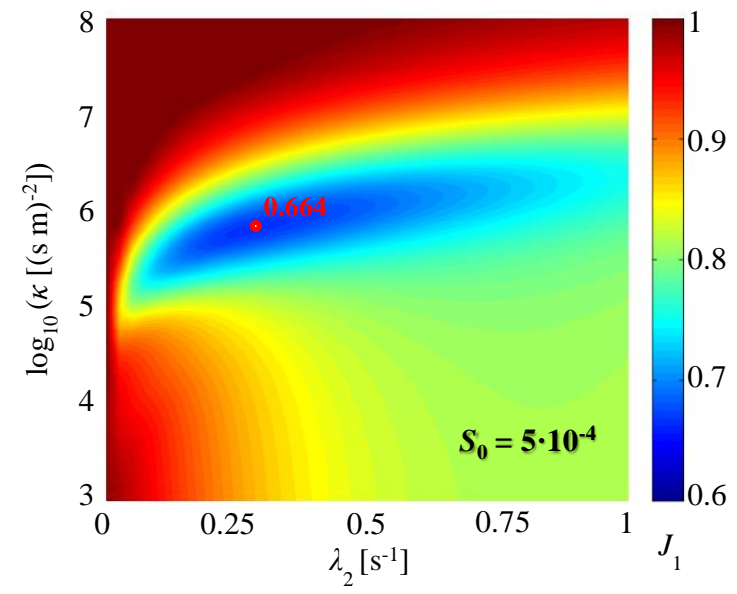

(d)

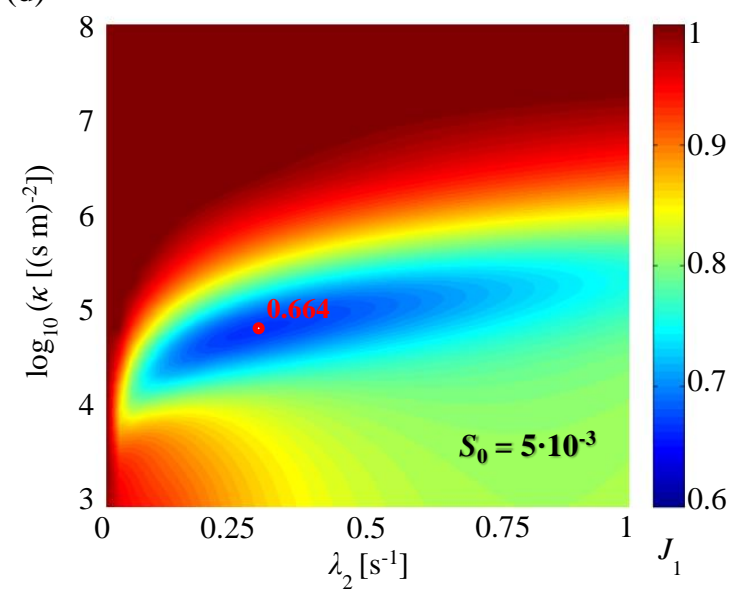

(e)

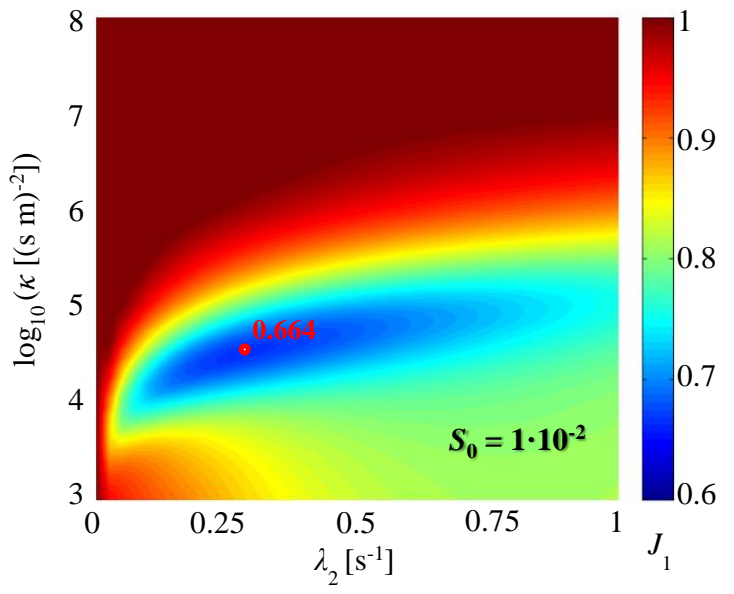

Figure 5: $J_{1}$ function with respect to the normalised nonlinear stiffness $\kappa$ and linear damping $\lambda_{2}$ varying $S_{0}\left[\left(\mathrm{~m} / \mathrm{s}^{2}\right)^{2} /(\mathrm{rad} / \mathrm{s})\right]$. 
(a)

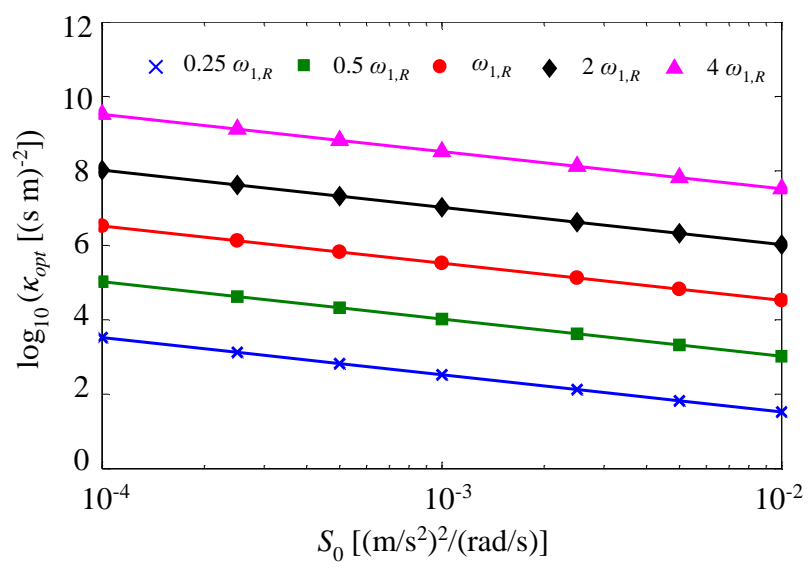

(b)

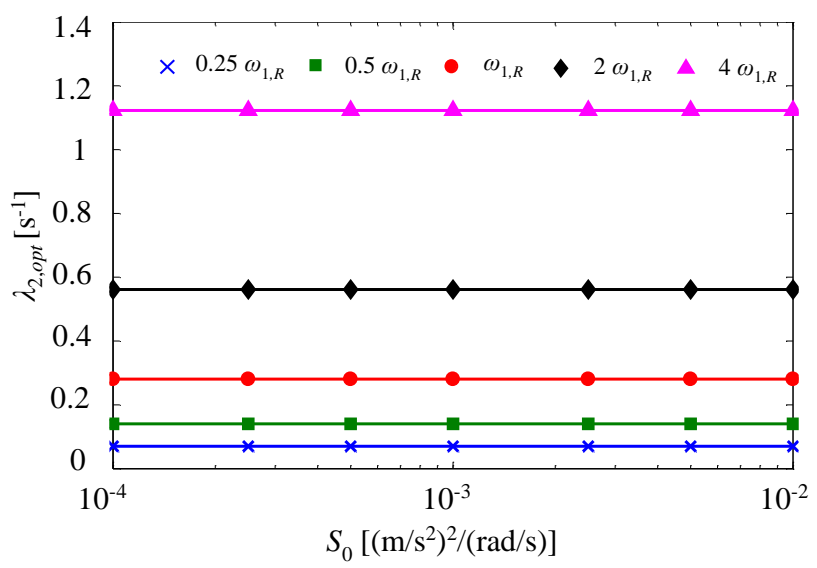

Figure 6: Optimal parameters of the NES obtained by MCS (symbols) and fitted curves (solid line) versus $S_{0}$ varying the main frequency $\omega_{1}\left(\zeta_{1}=\zeta_{1, R}, \varepsilon=0.05\right)$ :

a) normalised optimal NES stiffness $\kappa$; b) normalised optimal NES damping $\lambda_{2}$. 
(a)

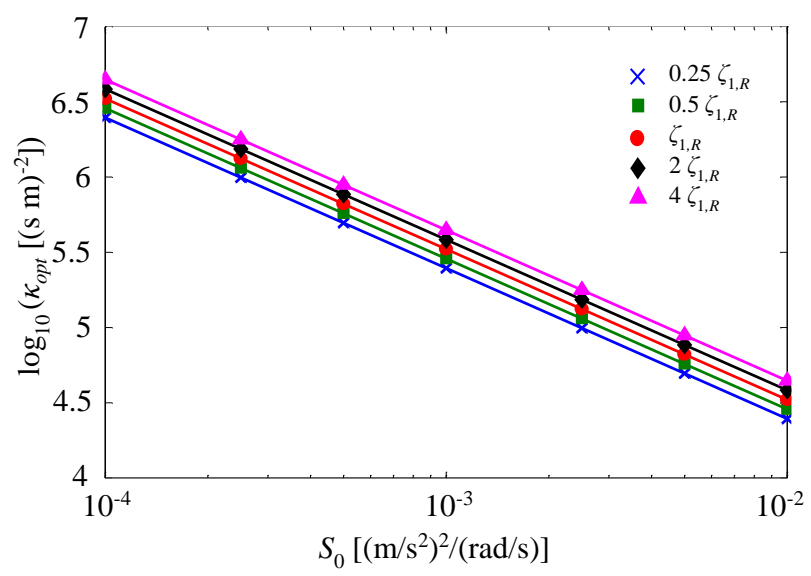

(b)

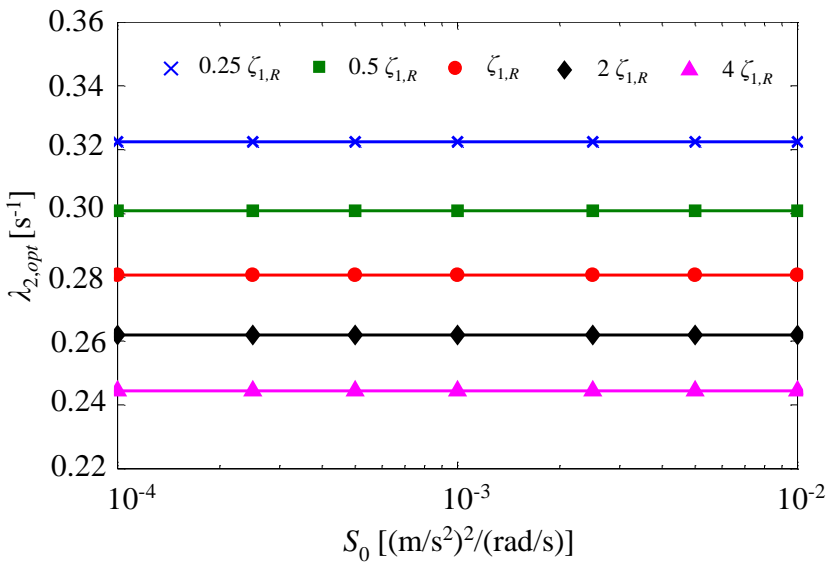

Figure 7: Optimal parameters of the NES obtained by MCS (symbols) and fitted curves (solid line) versus $S_{0}$ varying the main damping ratio $\zeta_{1}\left(\omega_{1}=\omega_{1, R}, \varepsilon=0.05\right)$ :

a) normalised optimal NES stiffness $\kappa$; b) normalised optimal NES damping $\lambda_{2}$. 
(a)

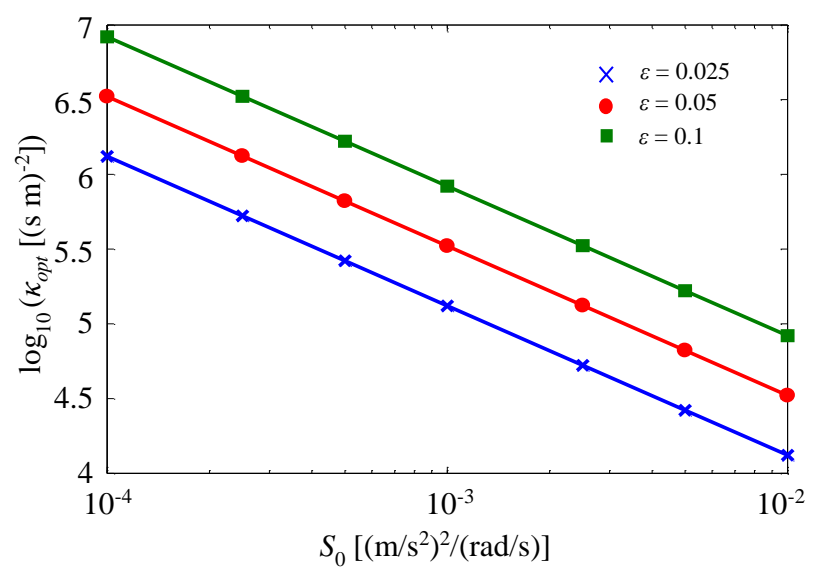

(b)

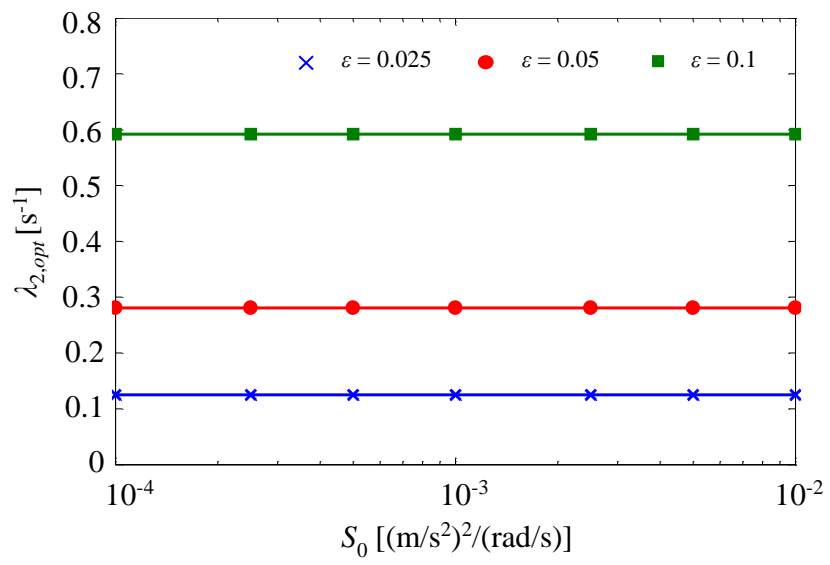

Figure 8: Optimal parameters of the NES obtained by MCS (symbols) and fitted curves (solid line) versus $S_{0}$ varying the mass ratio $\varepsilon\left(\omega_{1}=\omega_{1, \mathrm{R}}, \zeta_{1}=\zeta_{1, \mathrm{R}}\right)$ :

a) normalised optimal NES stiffness $\kappa$; b) normalised optimal NES damping $\lambda_{2}$. 

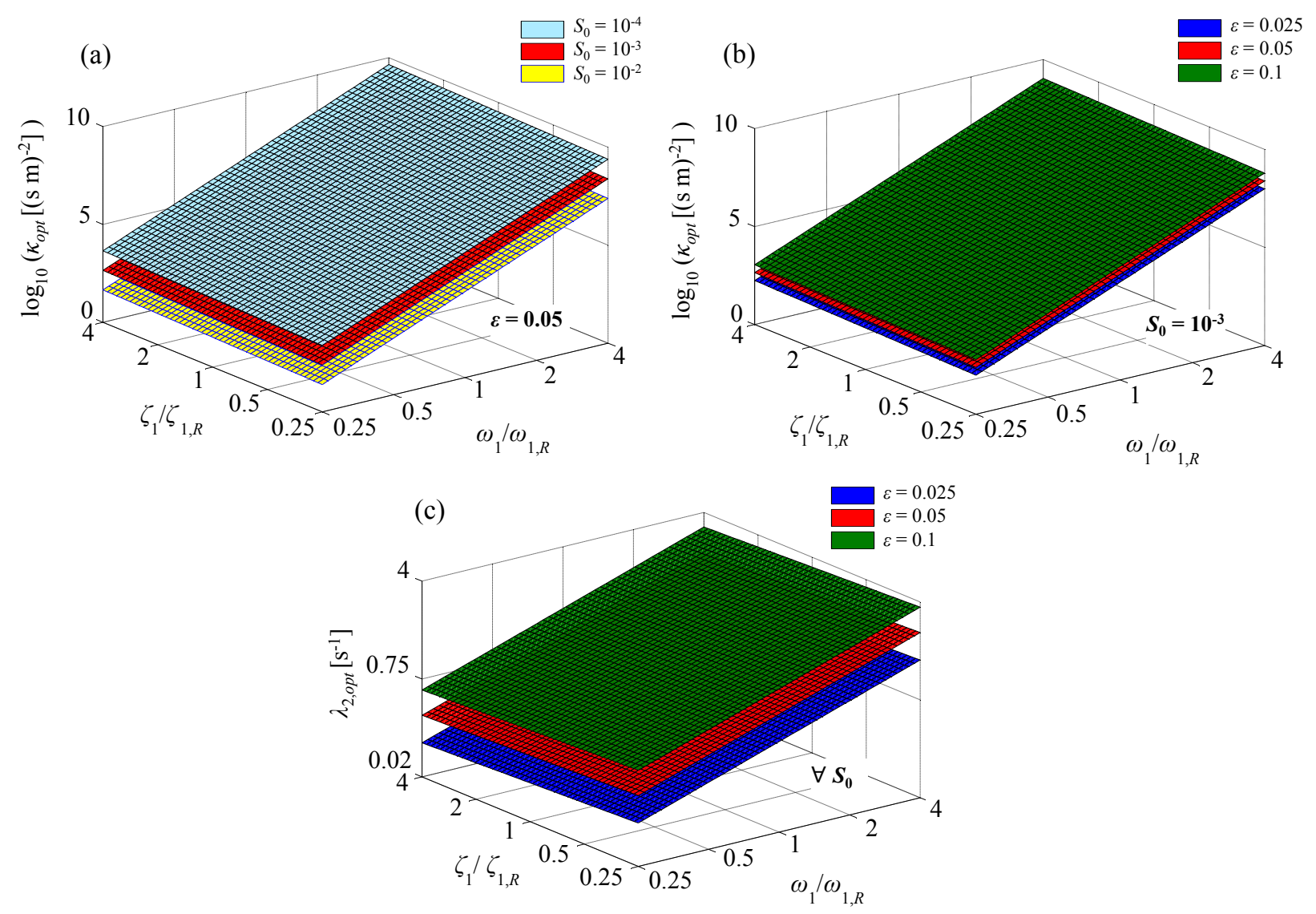

Figure 9: Optimal NES parameters for varying normalised frequency and damping of the primary structure: a) $\kappa_{\text {opt }}$ for fixed $\varepsilon$; b) $\kappa_{\text {opt }}$ for fixed $S_{0} ;$ c) $\lambda_{2, o p t}$. 


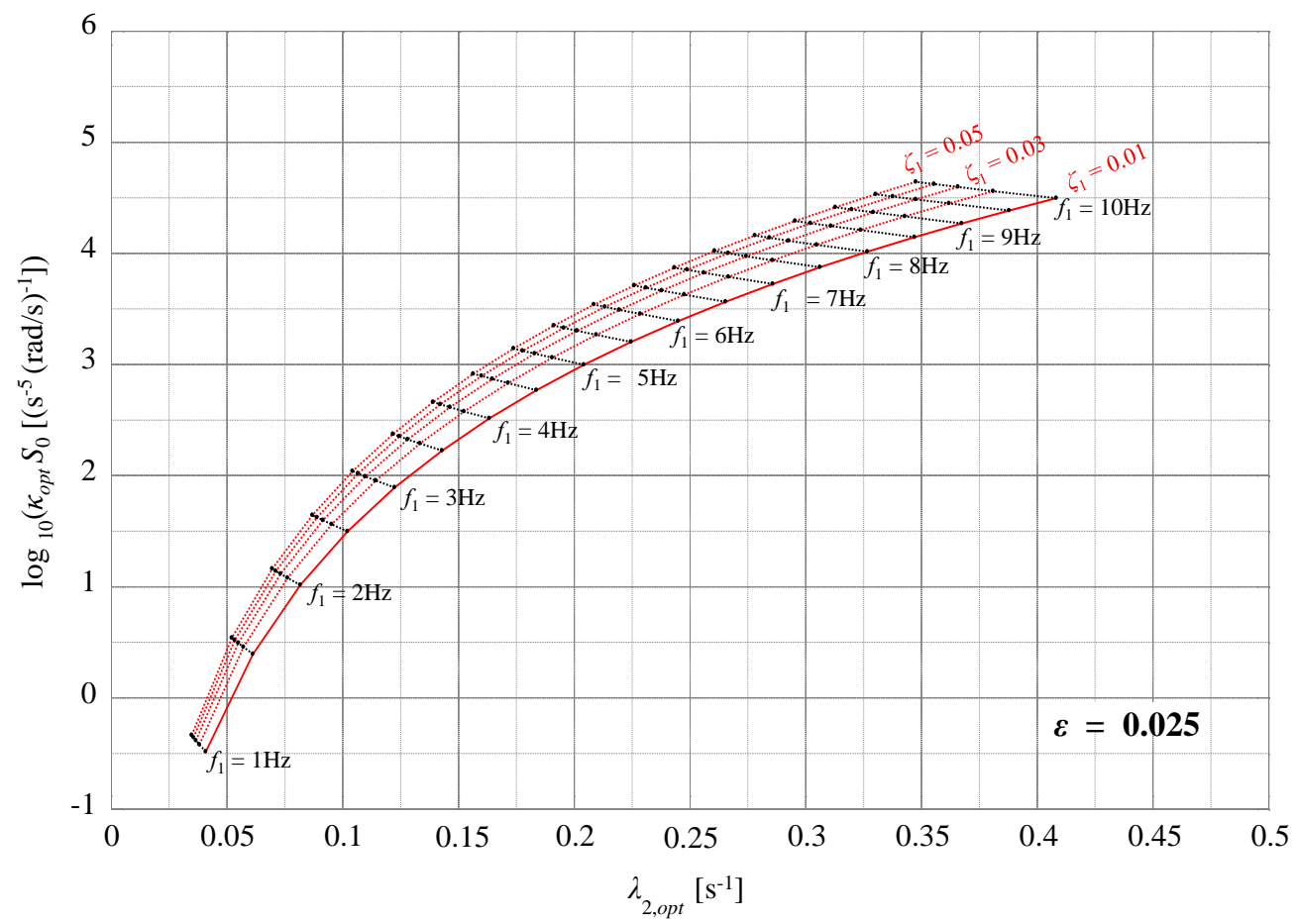

Figure 10: Design chart to determine the optimal NES parameters for different values of $\omega_{1}$ and $\zeta_{1}(\varepsilon=0.025)$. 


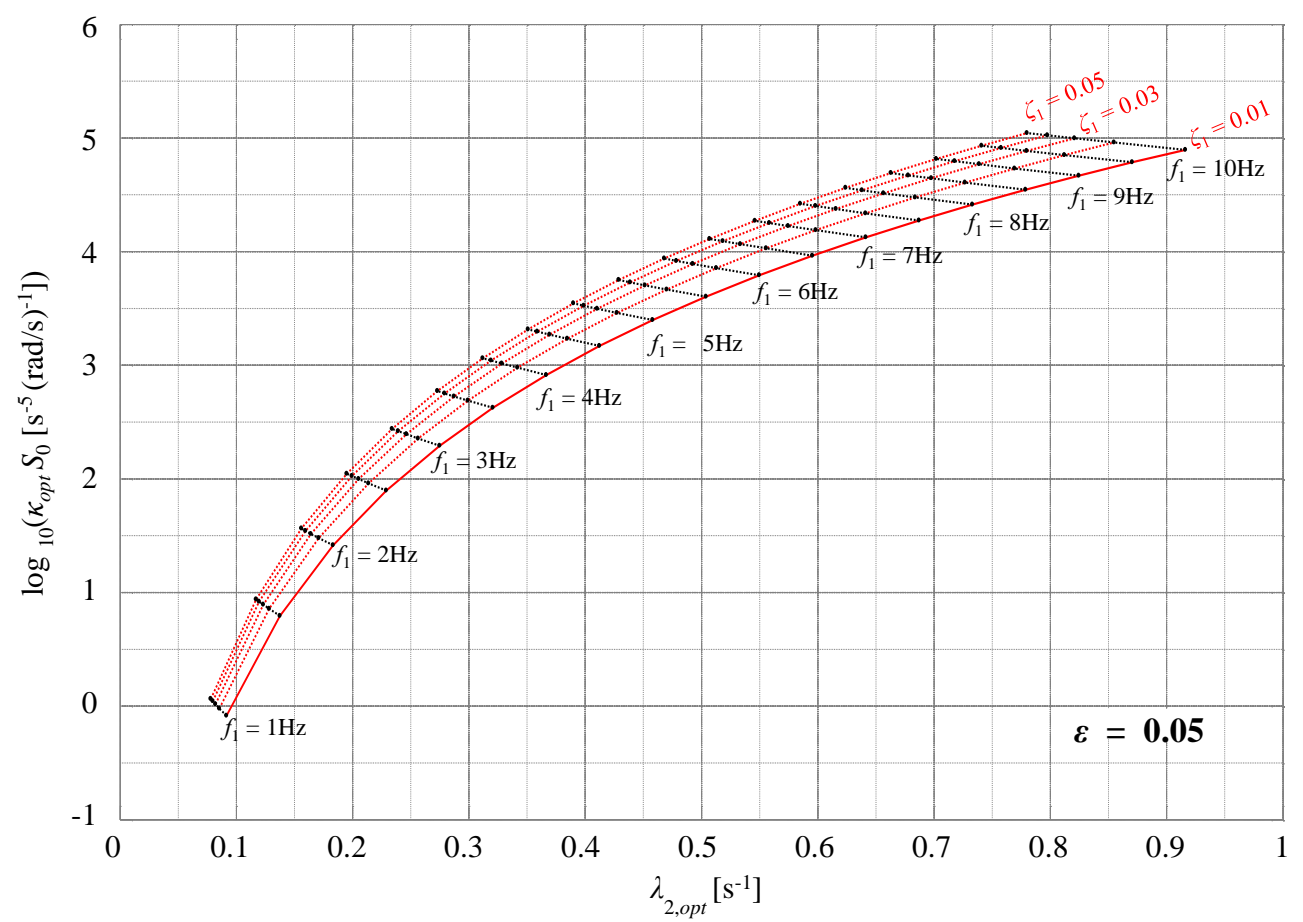

Figure 11: Design chart to determine the optimal NES parameters for different values of $\omega_{1}$ and $\zeta_{1}(\varepsilon=0.05)$. 


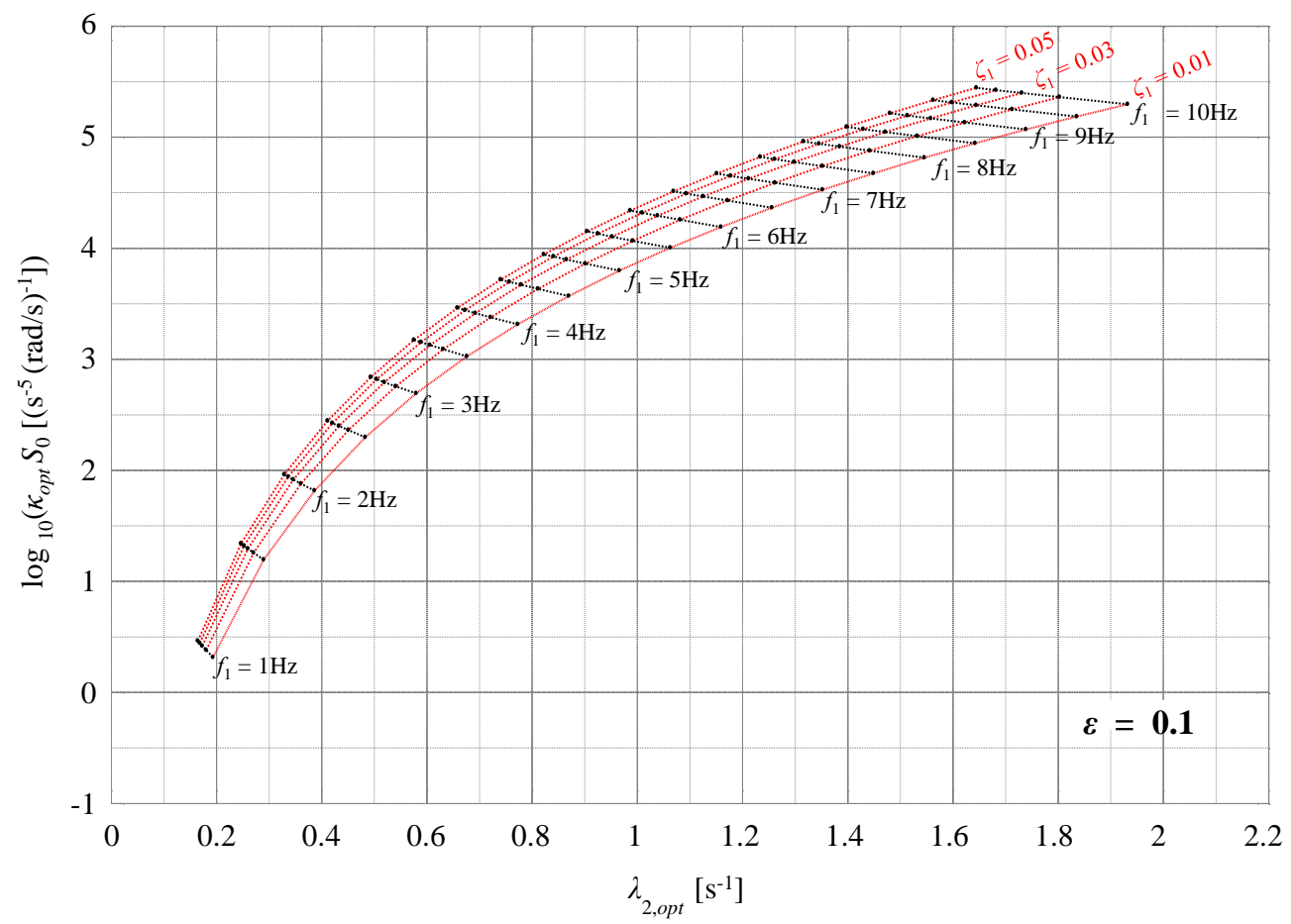

Figure 12: Design chart to determine the optimal NES parameters for different values of $\omega_{1}$ and $\zeta_{1}(\varepsilon=0.1)$. 
(a)
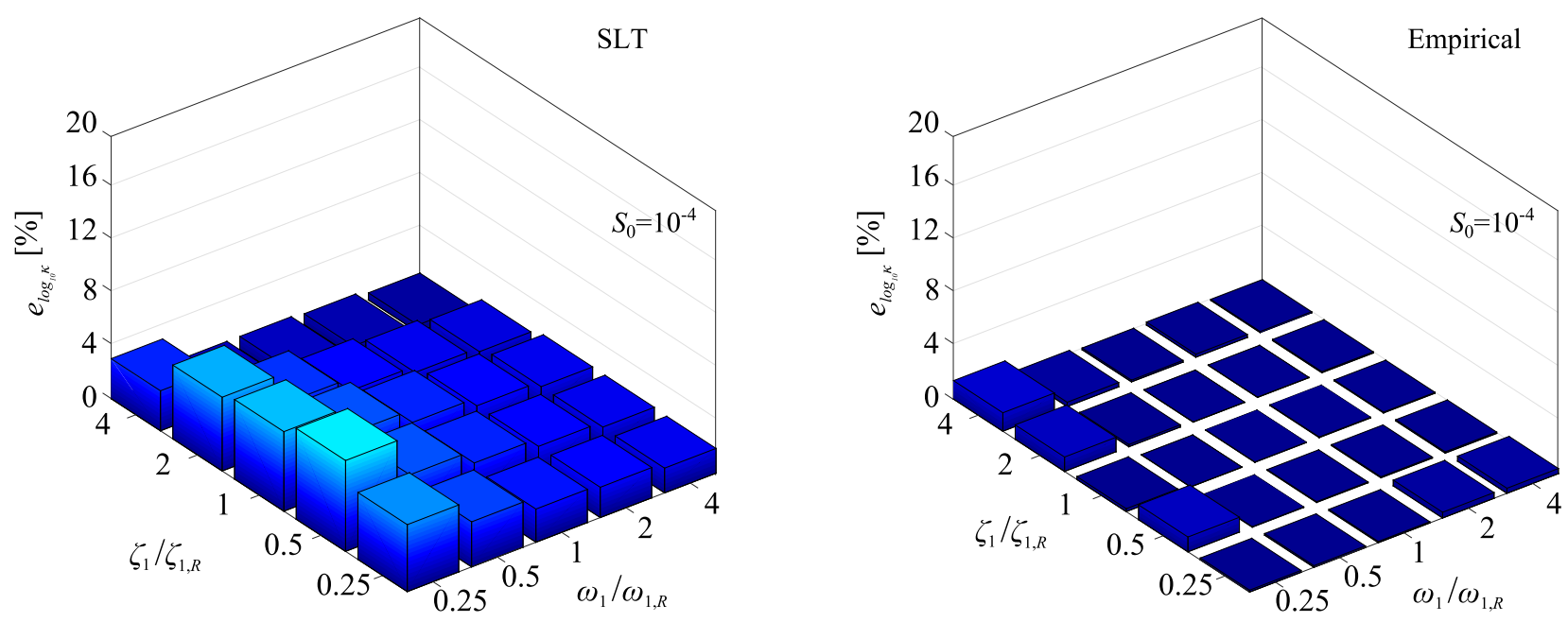

(b)
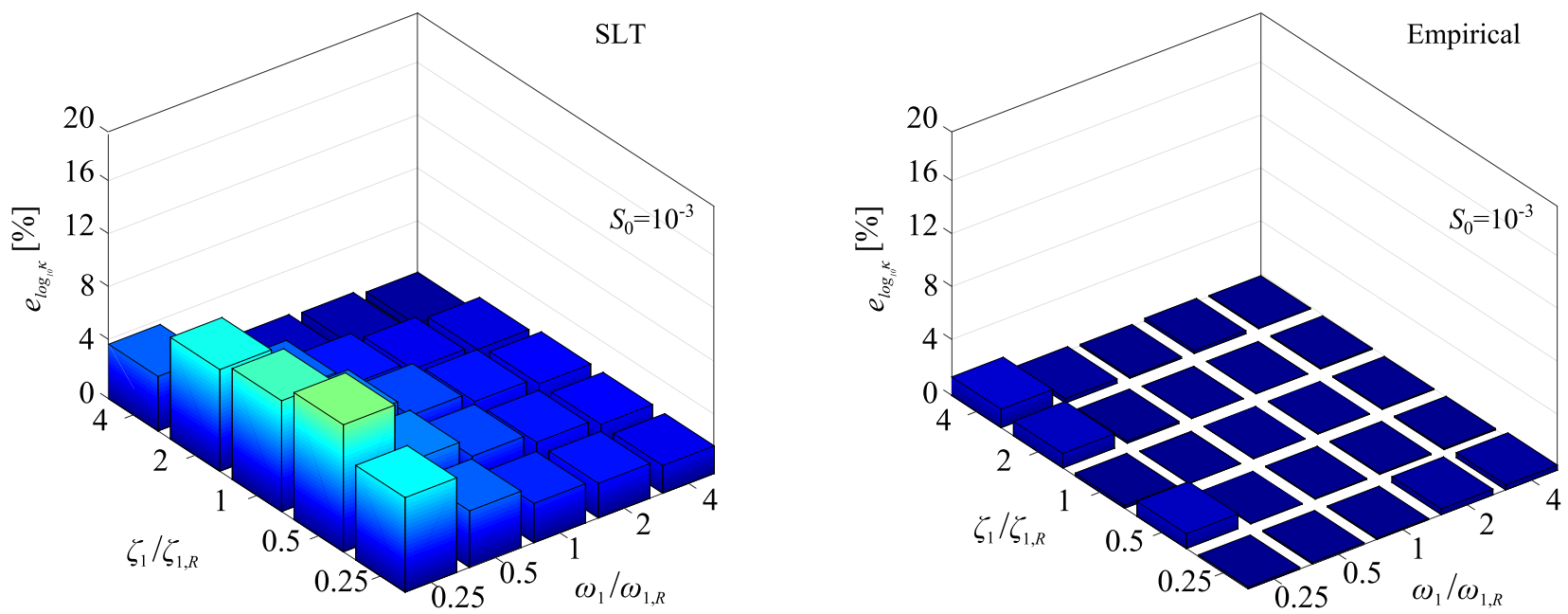

(c)
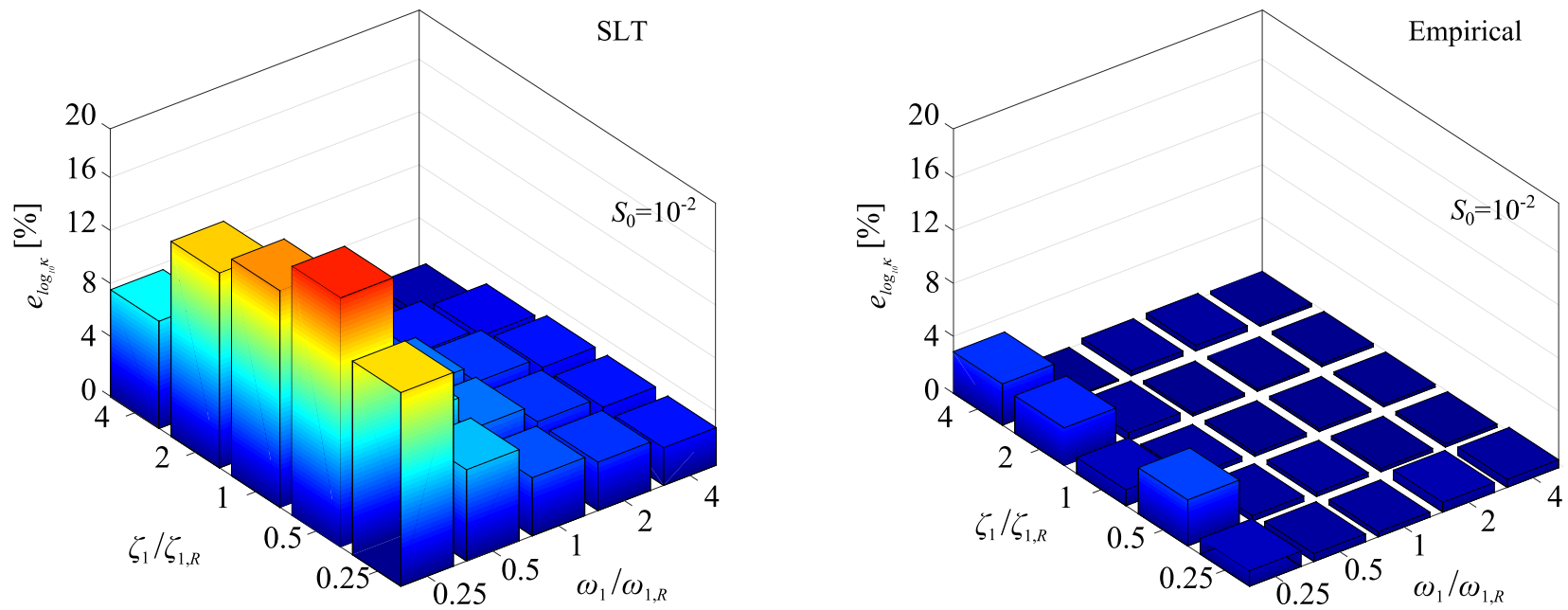

Figure 13: Percentage error commited in the evaluation of the NES optimal stiffness in logarithmic scale by using the SLT-based formula (20) and the empirical formula (28) for $\varepsilon=0.05$ and :

a) $S_{0}=10^{-4}\left(\mathrm{~m} / \mathrm{s}^{2}\right)^{2} /(\mathrm{rad} / \mathrm{s})$; b) $S_{0}=10^{-3}\left(\mathrm{~m} / \mathrm{s}^{2}\right)^{2} /(\mathrm{rad} / \mathrm{s})$; c) $S_{0}=10^{-2}\left(\mathrm{~m} / \mathrm{s}^{2}\right)^{2} /(\mathrm{rad} / \mathrm{s})$. 
(a)
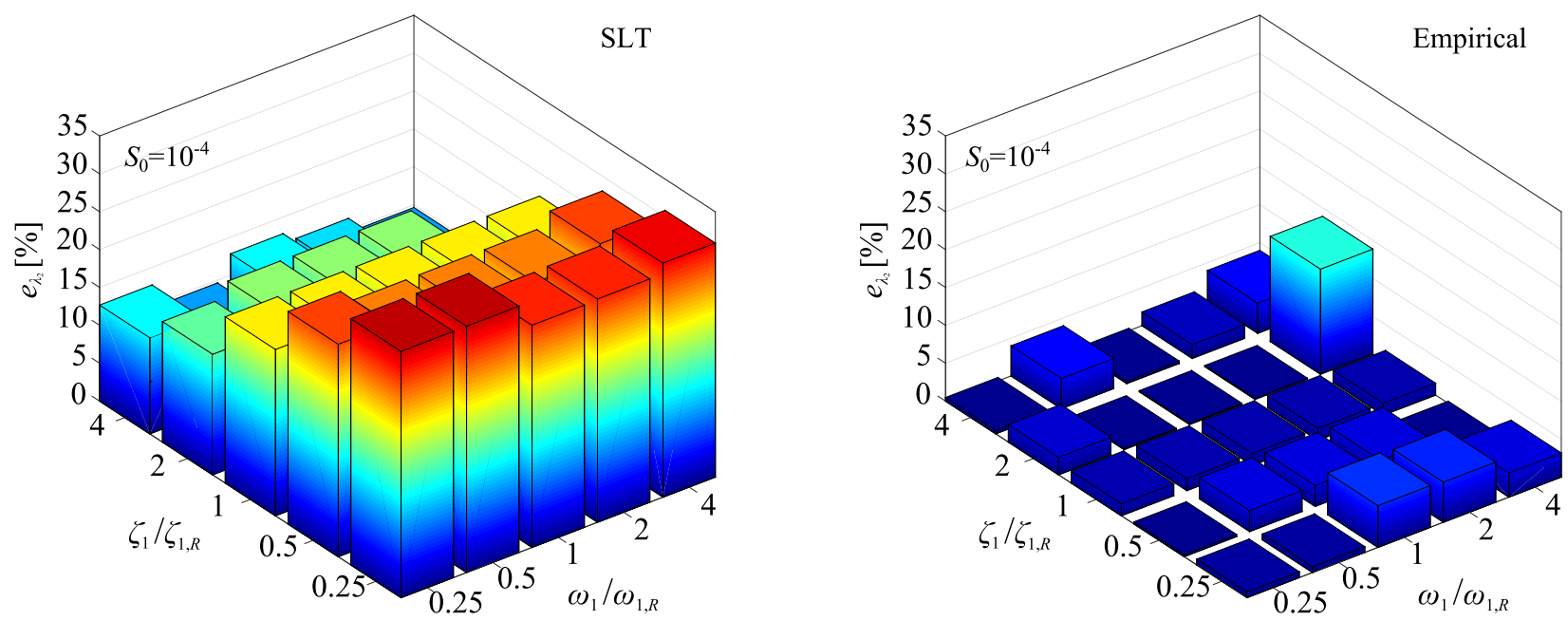

(a)
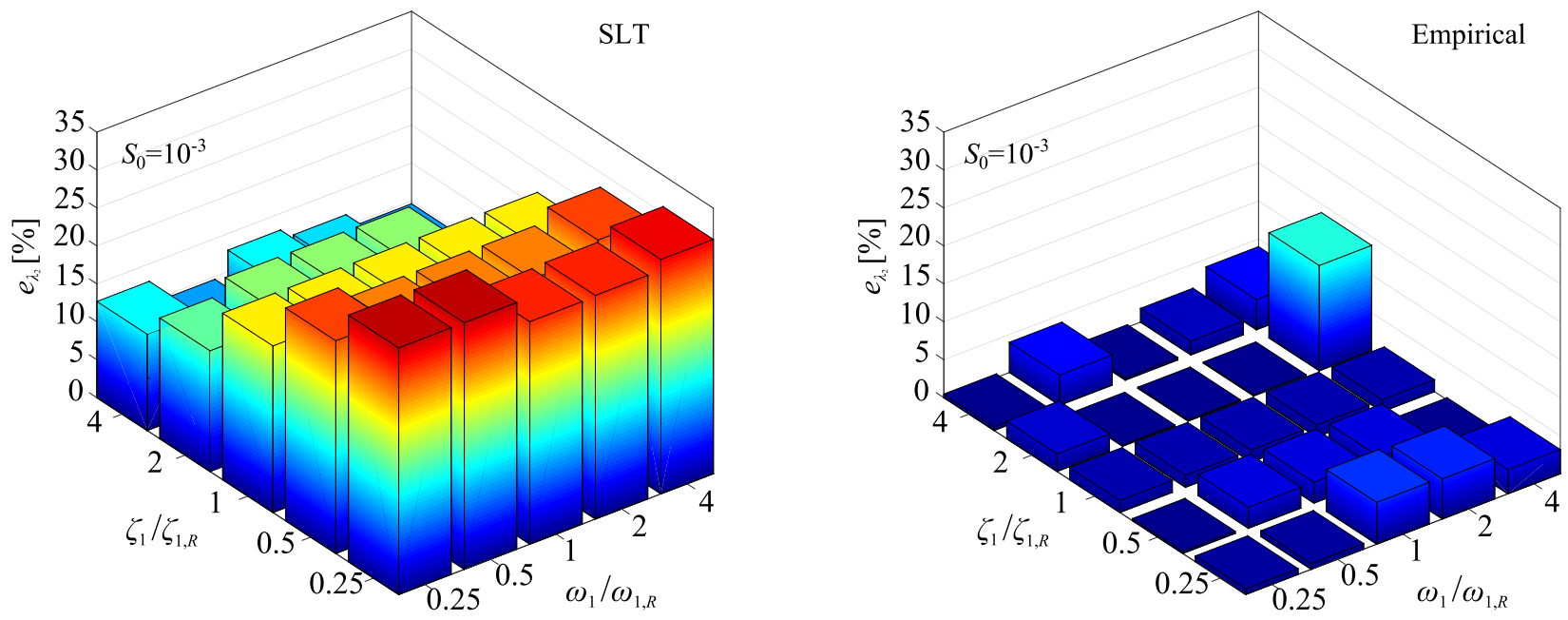

(a)
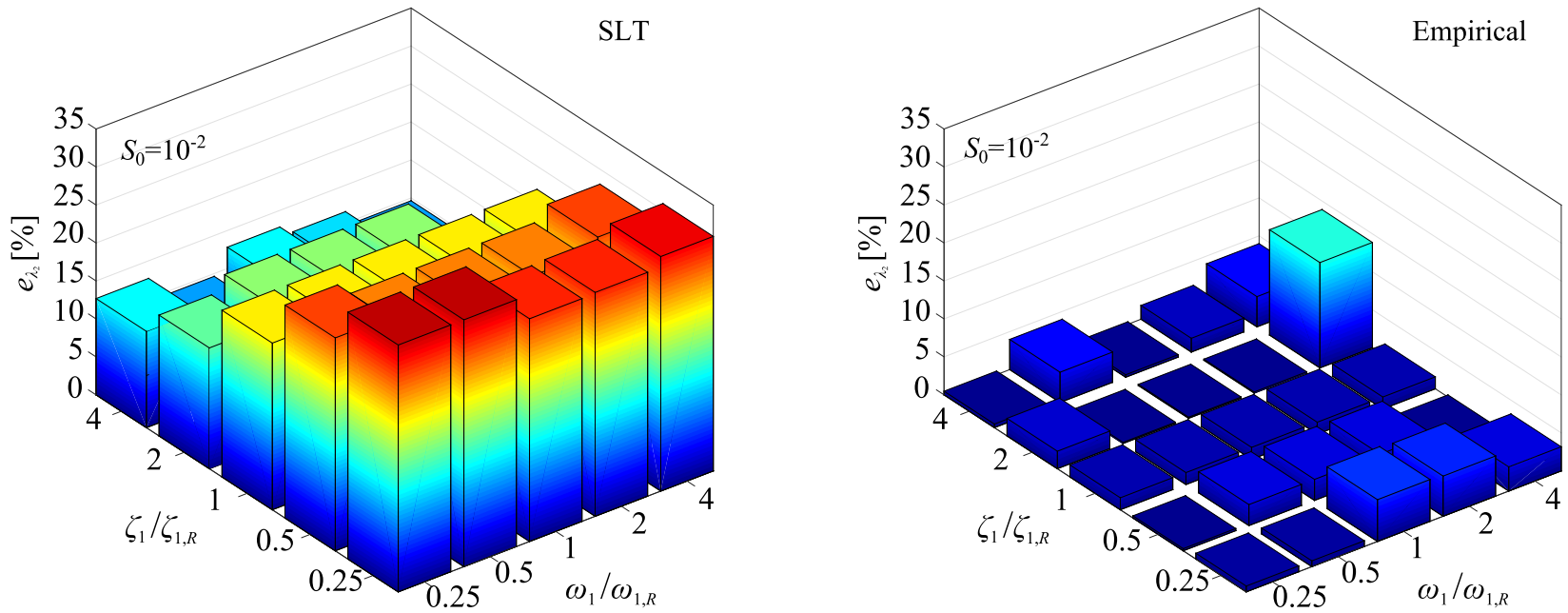

Figure 14: Percentage error commited in the evaluation of the NES optimal damping by using the SLT-based formula (21) and the empirical formula (29) for $\varepsilon=0.05$ and: a) $S_{0}=10^{-4}\left(\mathrm{~m} / \mathrm{s}^{2}\right)^{2} /(\mathrm{rad} / \mathrm{s})$;

b) $S_{0}=10^{-3}\left(\mathrm{~m} / \mathrm{s}^{2}\right)^{2} /(\mathrm{rad} / \mathrm{s}) ;$ c) $S_{0}=10^{-2}\left(\mathrm{~m} / \mathrm{s}^{2}\right)^{2} /(\mathrm{rad} / \mathrm{s})$. 
(a)
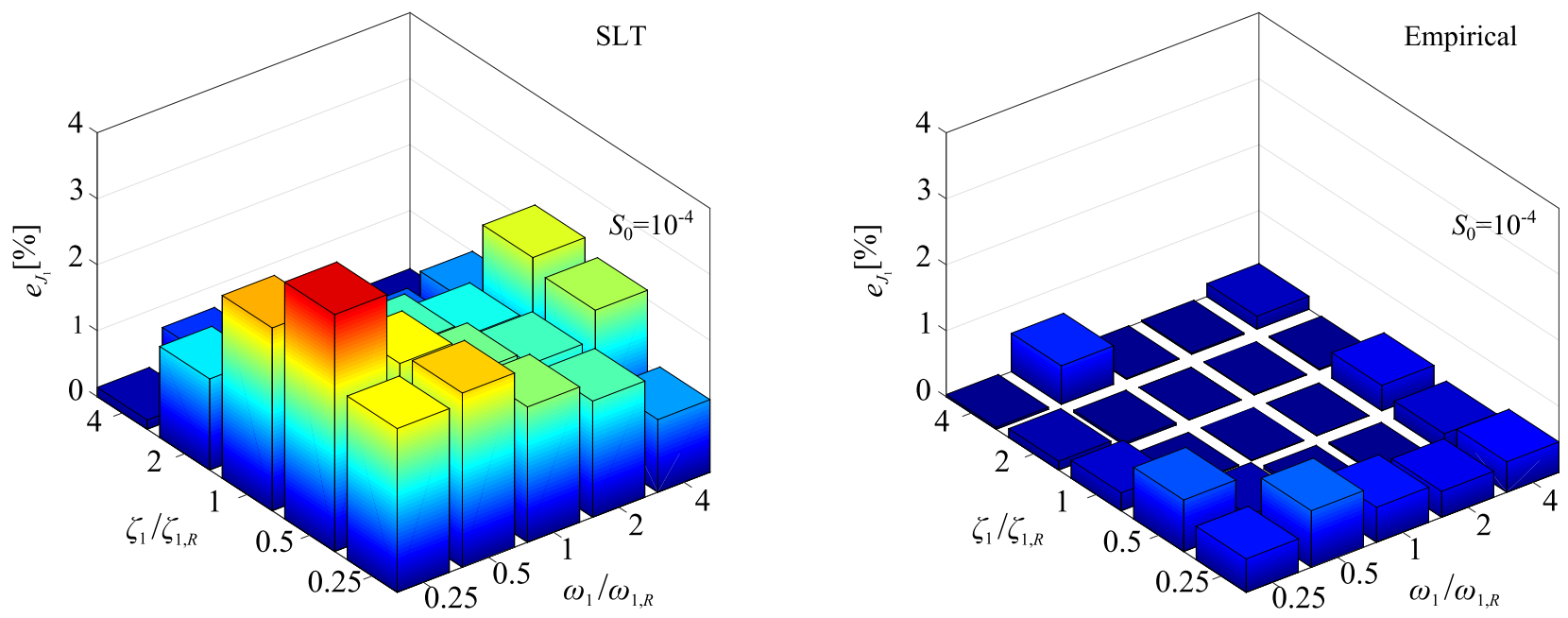

(b)
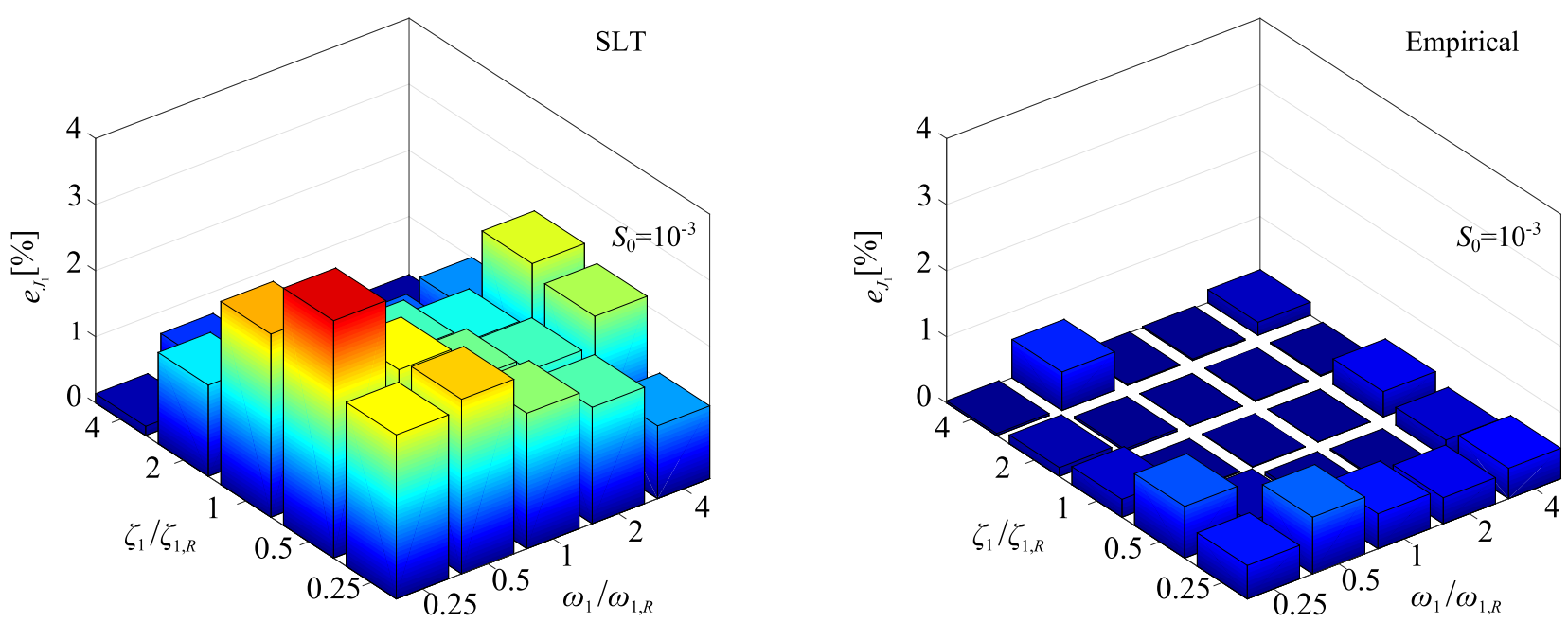

(c)
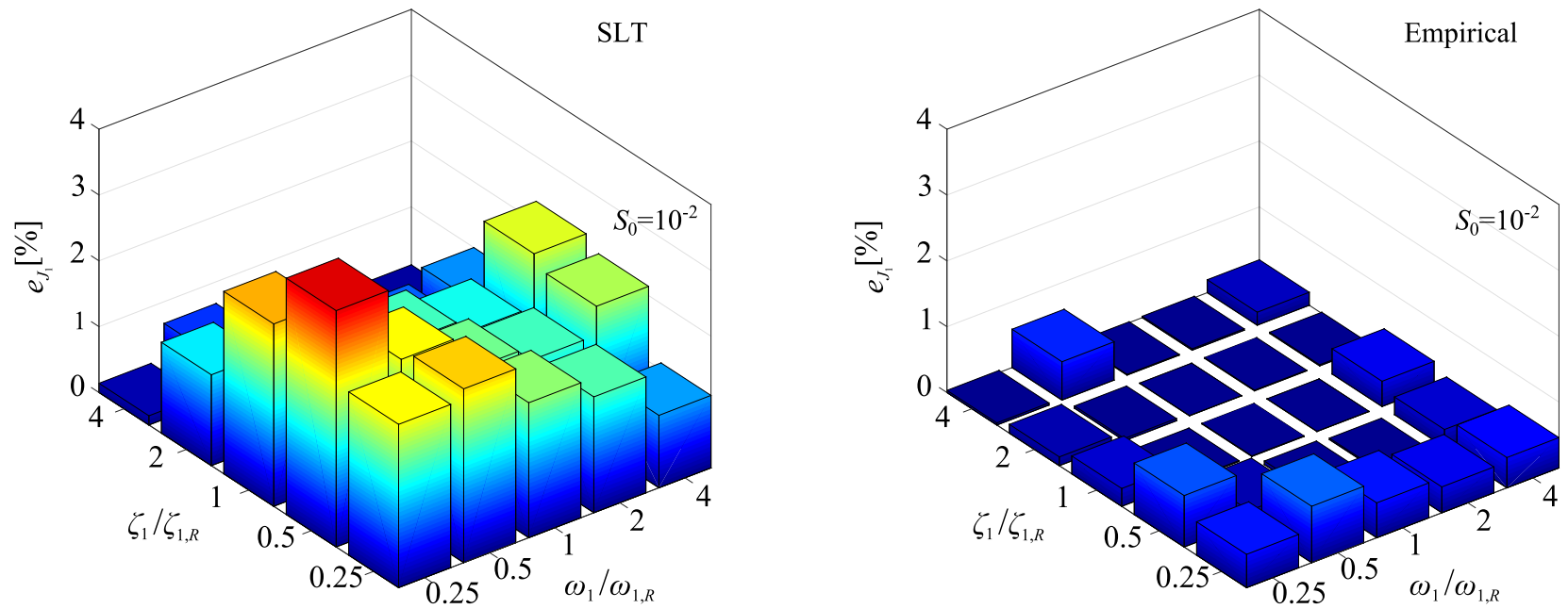

Figure 15: Percentage error commited on the estimation of the minimum of the $J_{1}$ function by using the SLT-based approach and the empirical approach for $\varepsilon=0.05$ and: a) $S_{0}=10^{-4}\left(\mathrm{~m} / \mathrm{s}^{2}\right)^{2} /(\mathrm{rad} / \mathrm{s})$; b) $S_{0}=10^{-3}\left(\mathrm{~m} / \mathrm{s}^{2}\right)^{2} /(\mathrm{rad} / \mathrm{s}) ;$ c) $S_{0}=10^{-2}\left(\mathrm{~m} / \mathrm{s}^{2}\right)^{2} /(\mathrm{rad} / \mathrm{s})$. 
(a)

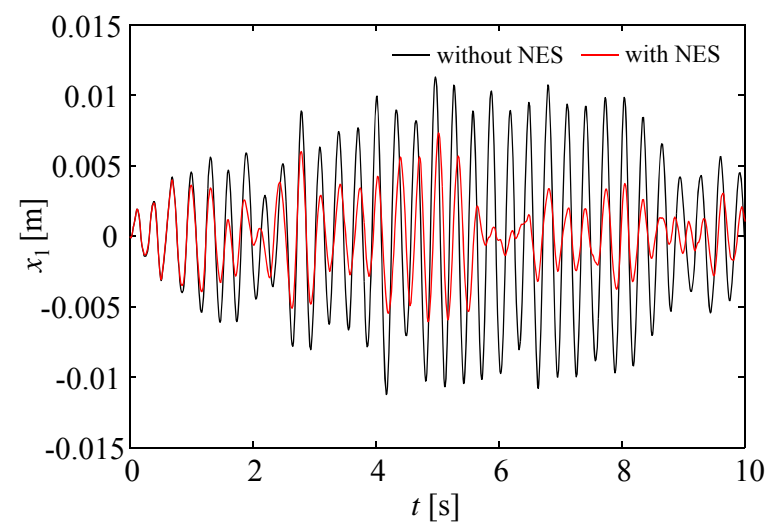

(c)

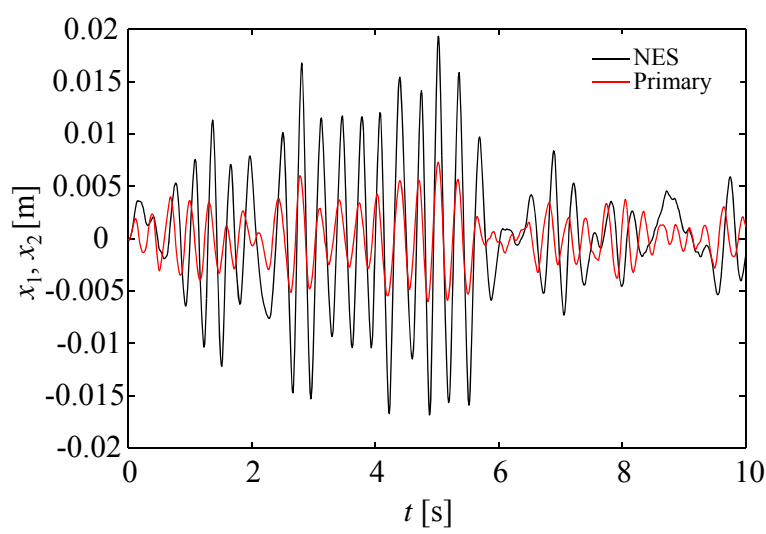

(e)

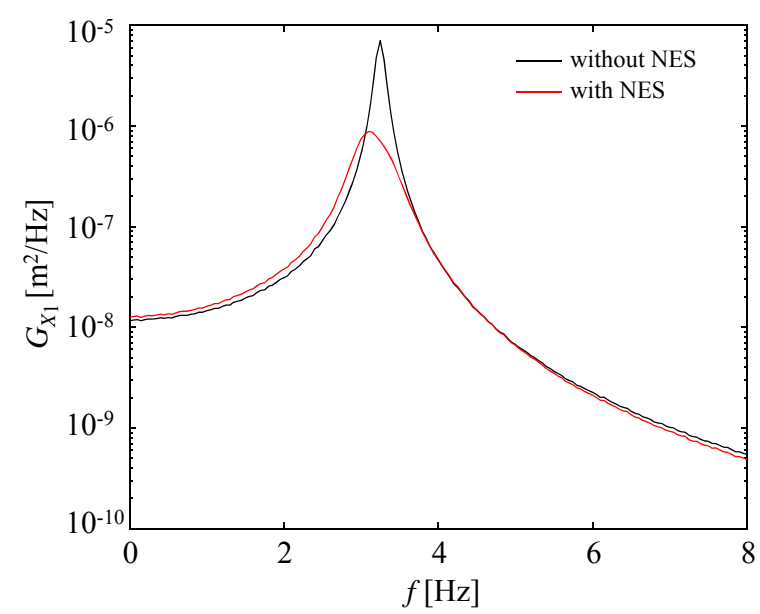

(b)

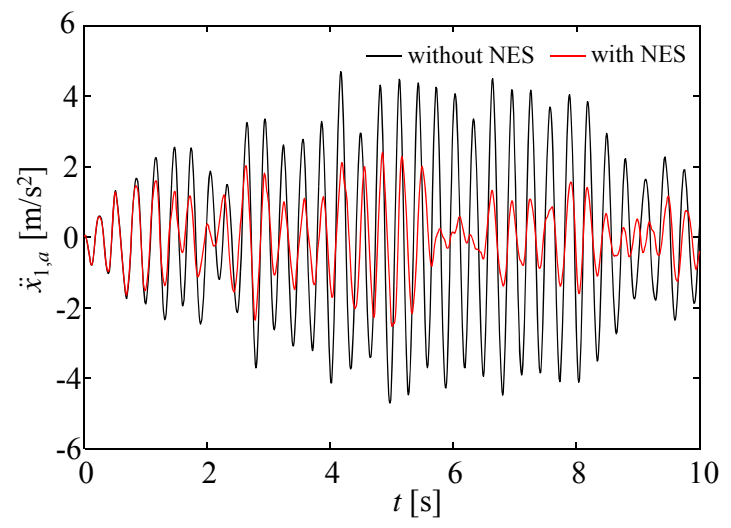

(d)

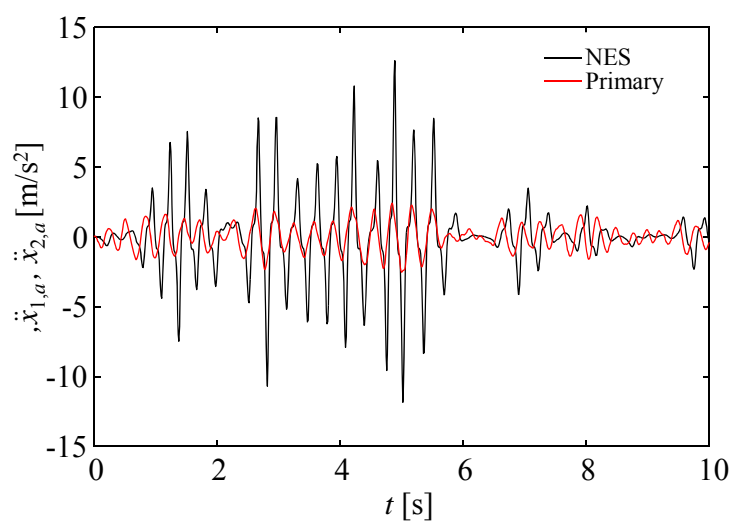

(f)

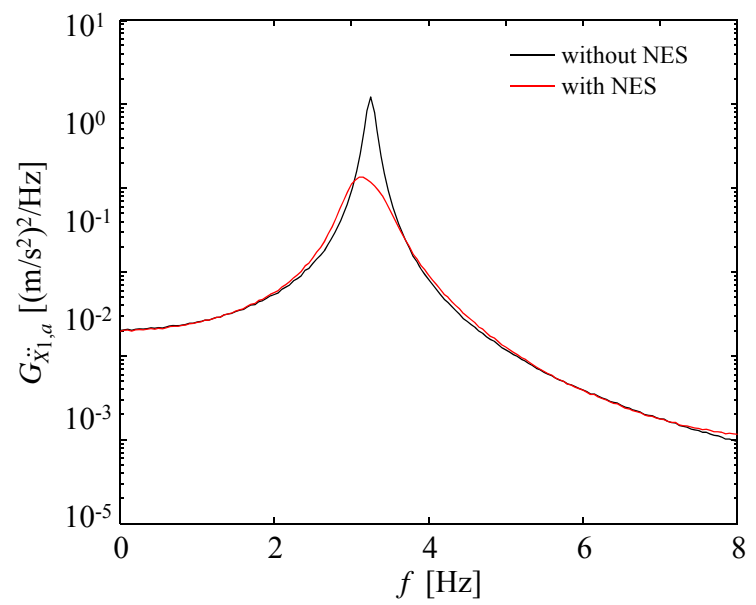

Figure 16: Comparison between the uncontrolled and controlled configurations in time and frequency domain: a) first $10 \mathrm{sec}$ of a sample of structural displacements process; b) first $10 \mathrm{sec}$ of a sample structural accelerations process; c) first $10 \mathrm{sec}$ of a sample of displacements process of the NES and primary structure; d) first $10 \mathrm{sec}$ of a sample of accelerations process of the NES and primary structure; e) PSD of displacements process; f) PSD of absolute accelerations process. 


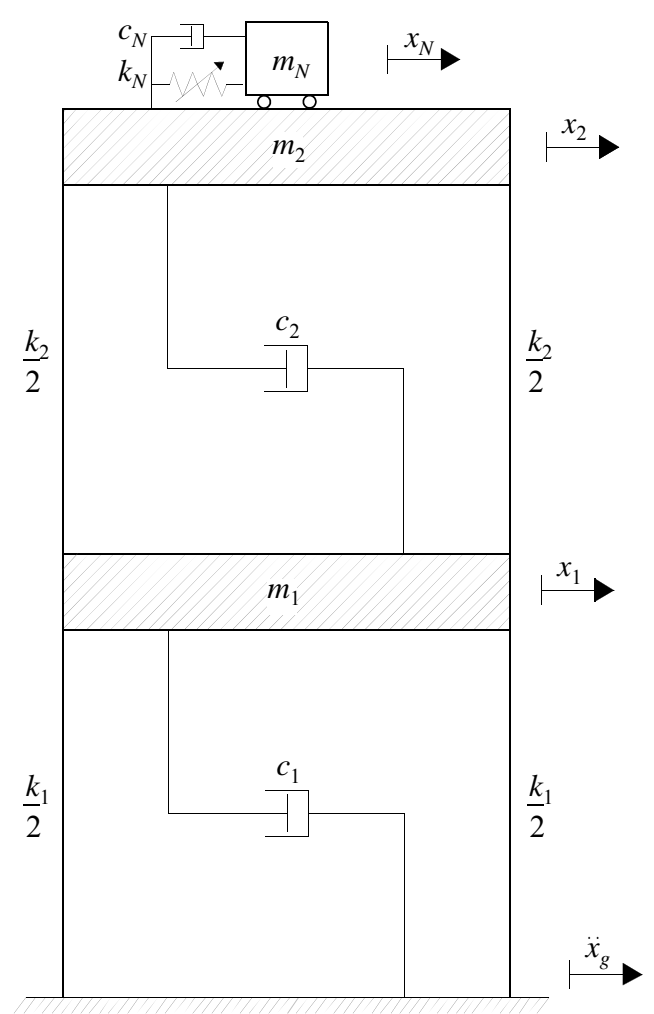

Figure 17: 2-DOF structure coupled with a NES. 
(a)

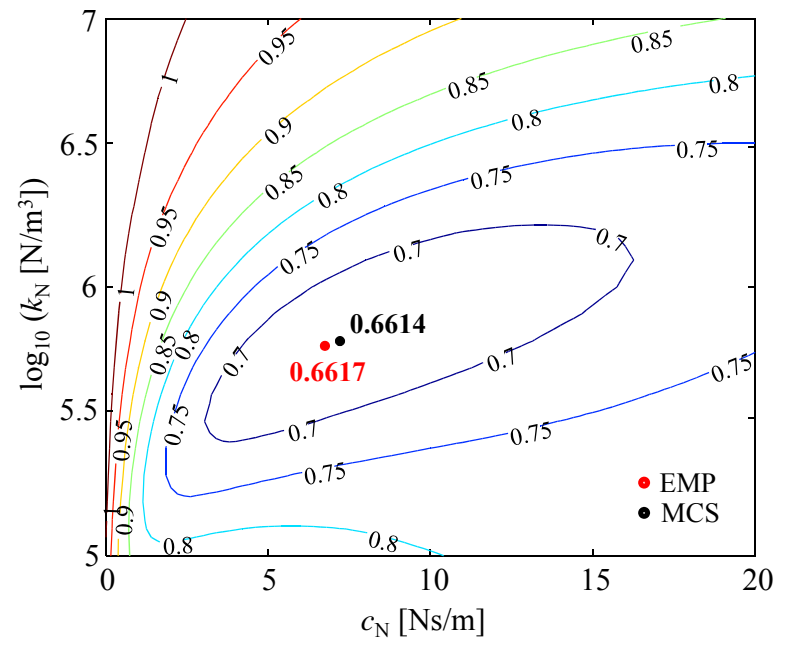

(b)

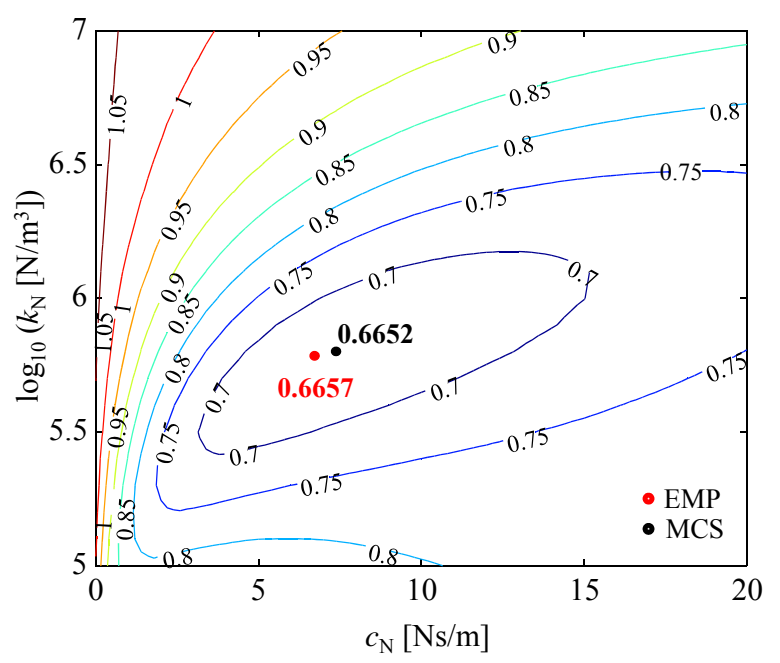

Figure 18: Objective functions and optimal NES parameters obtained by MCS (black dots) and by using the empirical formulae (red dots): a) $J_{1}$; b) $J_{2}$. 
(a)

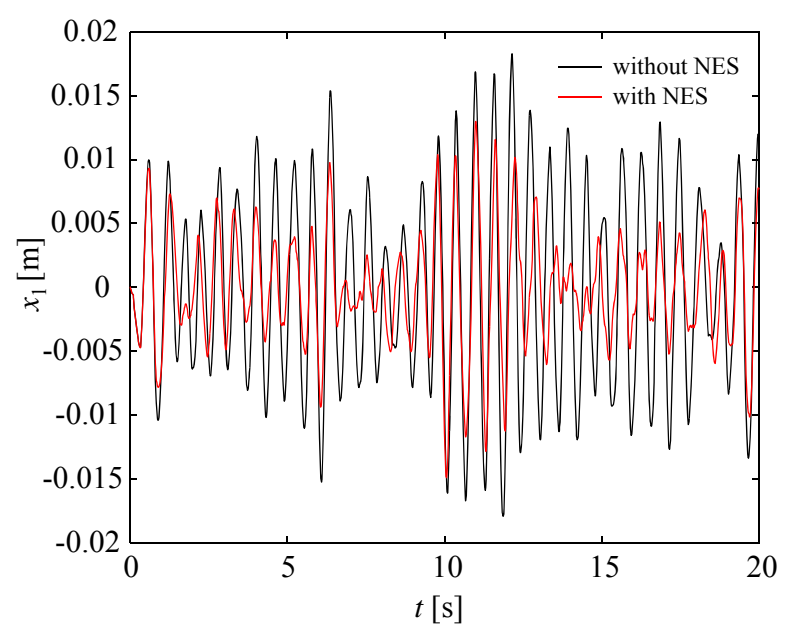

(c)

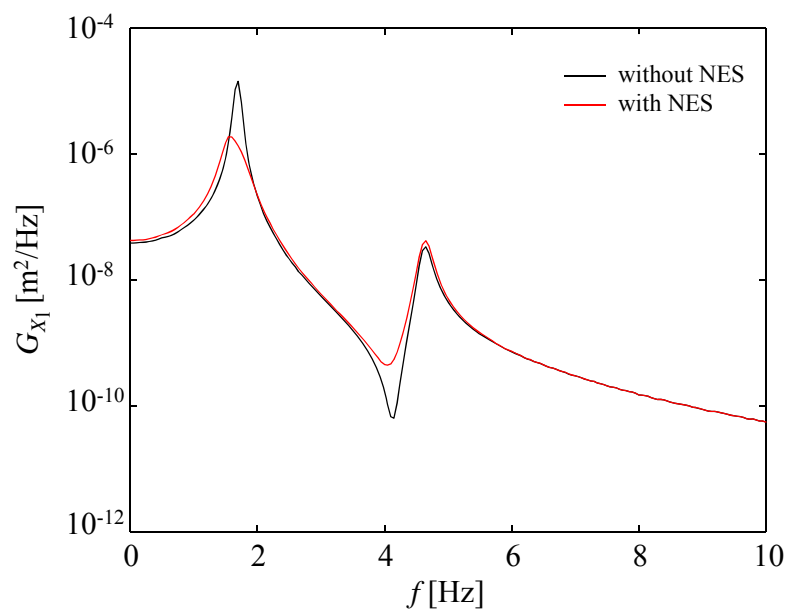

(b)

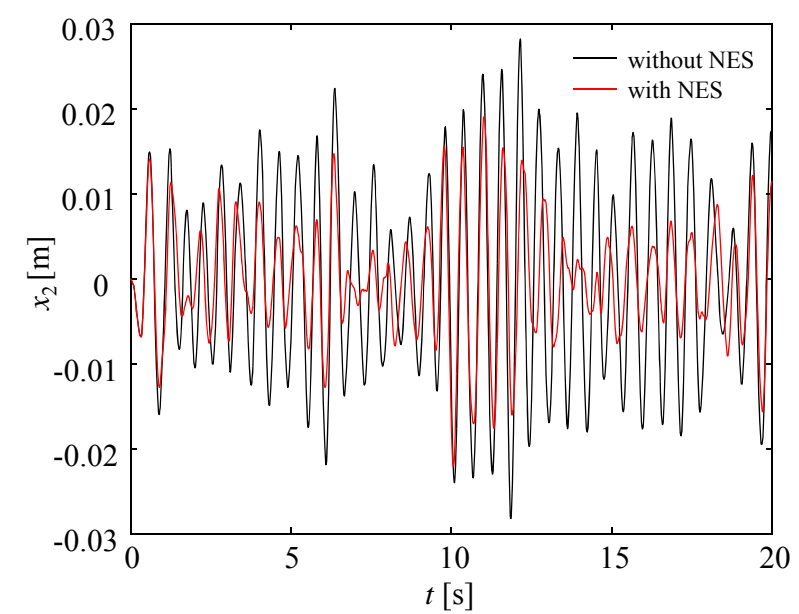

(d)

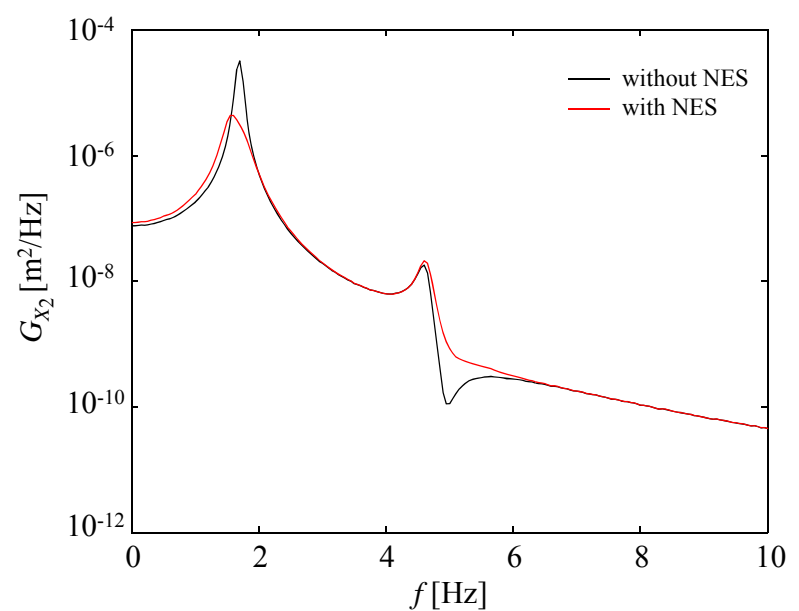

Figure 19: Comparison between the uncontrolled and controlled configurations in time and frequency domain: a) a sample of first storey displacements process; b) a sample of second storey displacements process; c) PSD of first storey displacements; d) PSD of second storey displacements. 